Meta

Journal des traducteurs

Translators' Journal

\title{
La fiction québécoise traduite en Espagne : une question de réseaux
}

\section{María Sierra Córdoba Serrano}

Volume 52, numéro 4, décembre 2007

La traduction et les études de réseaux

Translation and Network Studies

URI : https://id.erudit.org/iderudit/017696ar

DOI : https://doi.org/10.7202/017696ar

Aller au sommaire du numéro

\section{Éditeur(s)}

Les Presses de l'Université de Montréal

ISSN

0026-0452 (imprimé)

1492-1421 (numérique)

Découvrir la revue

Citer cet article

Córdoba Serrano, M. S. (2007). La fiction québécoise traduite en Espagne : une question de réseaux. Meta, 52(4), 763-792. https://doi.org/10.7202/017696ar
Résumé de l'article

Le but de cet article est d'analyser comment la fiction québécoise réussit à franchir les frontières linguistiques et culturelles et à se ménager un espace de réception dans les champs littéraires espagnol et catalan. Pour autant, l'existence de cet espace ne tient pas à la fonction spécifique que la fiction québécoise traduite remplit dans les champs littéraires cibles, mais plutôt à l'engagement personnel de certains agents des espaces sources et cibles, ainsi qu'à l'action déployée par les différents réseaux (des espaces sources, principalement) qui participent à l'exportation et à la promotion de la fiction québécoise en Espagne et en Catalogne. Le présent article s'attache à mettre au jour, à caractériser et à classifier les réseaux en question. 


\title{
La fiction québécoise traduite en Espagne: une question de réseaux
}

\author{
MARÍA SIERRA CÓRDOBA SERRANO \\ Université d'Ottawa, Ottawa, Canada \\ mcord059@uottawa.ca
}

\begin{abstract}
RÉSUMÉ
Le but de cet article est d'analyser comment la fiction québécoise réussit à franchir les frontières linguistiques et culturelles et à se ménager un espace de réception dans les champs littéraires espagnol et catalan. Pour autant, l'existence de cet espace ne tient pas à la fonction spécifique que la fiction québécoise traduite remplit dans les champs littéraires cibles, mais plutôt à l'engagement personnel de certains agents des espaces sources et cibles, ainsi qu'à l'action déployée par les différents réseaux (des espaces sources, principalement) qui participent à l'exportation et à la promotion de la fiction québécoise en Espagne et en Catalogne. Le présent article s'attache à mettre au jour, à caractériser et à classifier les réseaux en question.
\end{abstract}

\begin{abstract}
The goal of the article is to show how, in spite of the diffusion obstacles faced by NorthAmerican French culture, Quebec fiction has managed to cross linguistic and cultural borders and create its own space in the Spanish and Catalan literary fields. The existence of this space cannot be attributed, however, to any specific function that translated Quebec fiction fulfills in the target literary fields, but to the commitment of certain source and target agents, as well as the actions of the different institutional networks (especially those from the source space) participating in the export and promotion of Quebec fiction in Spain and in Catalonia. The present article brings to light, characterizes and classifies these networks.
\end{abstract}

\section{MOTS-CLÉS/KEYWORDS}

sociologie du champ littéraire, réseaux, fiction québécoise traduite, Espagne post-franquiste, Catalogne post-franquiste

\section{Introduction}

Un grand nombre de traductologues, notamment Toury (1995), soutiennent que la traduction est un fait de la culture cible, et qu'elle sert souvent d'outil stratégique que les agents du champ littéraire d'arrivée emploient pour renforcer leur propre prise de position dans ce champ (Casanova 2002; Bourdieu 2002, parmi d'autres), voire pour consolider les idéologèmes de la représentation sociale de la société cible (Brisset 1990). D’autres, comme Even-Zohar, énoncent des «lois d'interférence littéraire» (1990) telles que «A source literature is selected by prestige» ou «A source literature is selected by dominance» (1990: 59), cette dernière s'appliquant aux cas de domination coloniale. Si tous ces auteurs semblent suggérer que la traduction est initiée par la culture cible, les chercheurs adoptant des approches post-coloniales (Niranjana, Tymoczko, Venuti, etc.) ont contesté cette hypothèse, car dans les cas qu'ils étudient, le transfert est plutôt initié par la culture source qui, par l'entremise de la traduction, 
forge et consolide généralement les représentations propres au colonisateur. Notre article offre un exemple permettant de croire que l'hypothèse de ces chercheurs est valable, bien au-delà des contextes coloniaux ou post-coloniaux. Cette étude porte sur la fiction québécoise produite en français et traduite vers l'espagnol et vers le catalan en Espagne entre une date-clé de l'histoire espagnole contemporaine, 1975 (fin de la dictature franquiste), et 2004.

Le travail que nous présentons ici s'inscrit dans un projet de recherche en cours qui analyse non seulement la première phase du processus de transfert culturel $^{1}$ (l'objet de cet article), soit le rôle et les intérêts investis des acteurs ${ }^{2}$ publics ou semiprivés qui promeuvent la traduction de la fiction québécoise en Espagne, mais aussi le rôle d'autres agents (traducteurs, éditeurs, directeurs de collection, critiques littéraires, etc.) dans la sélection, la classification ${ }^{3}$, la production et la réception de la fiction québécoise traduite en Espagne. Le point de vue des agents sera complété par l'analyse des traductions proprement dites pour déterminer comment les trajectoires de ces agents, de même que d'autres facteurs institutionnels extralittéraires et certains aspects liés à l'état du champ littéraire cible, se manifestent dans les textes traduits en tant que tels.

La réflexion que nous amorçons ici vise à mettre au jour le processus d'exportation et de promotion de la fiction québécoise en Espagne, autrement dit la façon dont certains agents et acteurs "affiliés» aux institutions supra-littéraires ${ }^{4}$ ont développé des stratégies et des structures promouvant un intérêt pour la littérature québécoise en Espagne. L'étude de cette «cartographie institutionnelle» peut se révéler d'un grand intérêt pour diverses raisons. D’abord, parce que ce genre d'étude centrée sur le rôle des agents et des acteurs institutionnels ${ }^{5}$ est presque absent du domaine de la traductologie (à l'exception des travaux de von Flotow sur la diplomatie publique). Ensuite, parce que la tâche d'organisation et de préparation du terrain (de ces acteurs) pour l'arrivée des textes traduits dans les espaces culturels cibles est, à notre avis, primordiale: même si les éditeurs ont le dernier mot en ce qui concerne la sélection des œuvres à traduire, leur décision est susceptible d'être influencée par la mise en place des stratégies déployées par ces acteurs et agents institutionnels. Ces stratégies sont d'autant plus importantes si l'on considère que l'on est devant un champ culturel et littéraire périphérique, une «culture de jardin» comme l'appelait Gellner (1989: 77) qui, pour exister et pour être exportée, a besoin de ce genre d'appui institutionnel.

La question que pose cet article est donc la suivante: comment la fiction québécoise réussit-elle à se ménager un espace de réception dans les champs culturels espagnol et catalan ${ }^{6}$ ? En effet, si, en Espagne, comme ailleurs, les textes choisis pour la traduction provenant des Amériques sont surtout en anglais, et si les traducteurs et les éditeurs de littérature en français ont la possibilité de choisir et connaissent mieux - entre autres, pour des raisons évidentes de proximité géographique - le corpus littéraire hexagonal, par quelles stratégies le Québec peut-il manifester son existence en Espagne? Qu'est-ce qui motive dans ce pays le choix de faire traduire des textes québécois?

Pour répondre à ces questions, deux modèles théoriques, qui mettent l'accent sur les agents plutôt que sur les textes, seront mobilisés: d'une part, celui de la sociologie du champ littéraire élaboré par Bourdieu, et d'autre part (mais en tant que complément du premier), celui de la sociologie des réseaux de Latour et Callon et du courant de recherche s'y rapportant. 


\section{Délimitation du corpus : la «fiction québécoise»}

Notre corpus compte des œuvres de fiction, y compris pour la jeunesse, et s'élève à un total de 77 traductions, dont la liste est présentée en annexe. Établir des critères pour délimiter pareil corpus présente plusieurs obstacles et soulève des questions: est-ce que par «fiction québécoise» on entend une œuvre écrite par un auteur québécois? par un auteur qui réside au Québec? une œuvre publiée par un éditeur québécois? qui parle du Québec? La définition de ce corpus pose un problème qui, en réalité, n'est pas propre à la fiction québécoise, mais à tout corpus issu d'une littérature relativement jeune, définie par des critères culturels plutôt que strictement linguistiques et à la fois centre et périphérie. En effet, dans le cas du champ québécois, plusieurs facteurs sont à considérer: d'une part, la relation ambivalente par rapport au champ français, "banque centrale symbolique» (Casanova 1999), et donc source de légitimation, en même temps qu'espace à partir duquel se démarquer. Ce champ québécois, cependant, constitue également un «centre» par rapport à d'autres littératures périphériques franco-canadiennes. À cette situation complexe s'ajoute l'arrivée sur la scène littéraire québécoise d'auteurs allophones écrivant en français et faisant partie du champ littéraire québécois. La multipositionnalité des écrivains dans plus d'un champ semble être une constante dans le cadre littéraire québécois, d'où la complexité de délimiter notre corpus.

C'est ici que la notion de "champ littéraire» et de «trajectoire», empruntée à Bourdieu, se révèle particulièrement utile. Bourdieu (1998) conçoit les œuvres comme des «prises de position» dans un champ, et les «carrières» des écrivains comme des trajectoires à l'intérieur de celui-ci. C'est la notion de «trajectoire» (Bourdieu 1998), c'est-à-dire la série de positions que les agents occupent dans un champ ou dans des champs différents qui nous a permis de délimiter notre corpus. Ainsi, nous avons retenu tous les textes de fiction produits par les écrivains d'expression française dont la trajectoire est intimement liée au champ littéraire québécois et qui ont pris activement position dans ce champ. Nous avons donc inclus non seulement les auteurs canadiens d'origine québécoise (c'est-à-dire les œuvres d'écrivains qui sont nés, vivent, écrivent et publient au Québec, même si, à un moment donné, ils choisissent ensuite, comme Anne Hébert, de vivre dans un autre pays et même si certaines de leurs œuvres ont été publiées ailleurs), mais aussi les auteurs d'origine non québécoise, pourvu que ces auteurs aient pris position dans le champ littéraire québécois, en publiant des articles dans des revues littéraires ou dans des journaux québécois, en assistant à des colloques littéraires au Québec, en étant membre de l'Union des écrivaines et des écrivains québécois, etc. Nous avons aussi considéré les œuvres d'auteurs publiés hors Québec mais qui auraient commencé leur activité d'écrivain au Québec, comme par exemple Nelly Arcan et Folch-Ribas. Enfin, figurent les œuvres d'auteurs non canadiens, qui font toutefois partie intégrante du patrimoine littéraire québécois, comme, par exemple, Maria Chapdelaine de Louis Hémon.

\section{Un champ peuplé d'agents coopérant selon une logique de réseau}

Dans In Search of a Theory of Translation (1980), Toury affirme que les textes traduits sont «facts of one language and one textual tradition only: the target's» (1980:16). Plus tard, il modifie légèrement cette affirmation: «translations are facts of target 
cultures; on occasion facts of special status [...] but of the target culture in any event " (1995: 26). Nombre de traductologues insistent encore sur cette vision téléologique selon laquelle la traduction viendrait toujours combler un vide ou remplir une fonction (attribuée a priori) dans la culture cible. Or, comme l'indique Bourdieu luimême: «Lattention exclusive aux fonctions conduit à ignorer la logique interne des objets culturels, leurs structures en tant que langages; mais, plus profondément, elle conduit à oublier les groupes qui produisent ces objets» (1994: 67).

Pour notre part, nous n'insisterons pas non plus sur les fonctions des traductions dans le champ cible, mais plutôt sur le rôle des différents agents et acteurs institutionnels des deux communautés source et cible - chacun ayant des motivations différentes - dans la création et la négociation d'un espace de réception pour ces traductions au sein des espaces culturels cibles. Comme l'affirme Buzelin: "The hypothesis that translation is a response to a need is not wrong, but [...] it leaves unexplained, the negotiations, struggles, tensions and basically all the strategies by which a foreign literature [...] makes its way and creates its own space in a domestic literary field» (2005: 207-208).

En effet, bien que le paradigme dominant en traductologie, les études descriptives, ait eu le mérite de placer la traduction dans le champ plus large de la culture, et bien que plus tard, après le virage culturel des années 1990 et le courant traductologique postcolonial qui a suivi, il ait porté plus d'attention aux rapports de pouvoir entre les communautés culturelles sources et cibles, les agents et les institutions participant à l'entreprise de la traduction et y investissant un certain intérêt ont toutefois été presque absents de ces travaux (Hermans 1999 et Pym 1998, parmi d'autres). Pour corriger cette lacune, vers la fin des années 1990, on observe la prolifération de travaux d'orientation plus sociologique qui amorcent le virage vers l'agent ou plutôt vers les agents, et s'inspirent en grande partie du modèle de Bourdieu?

Mener une analyse bourdieusienne revient avant tout à étudier les enjeux sociaux de la traduction en tant que production culturelle diffusée dans un espace social spécifique (le champ cible), qui est un espace de lutte (ou de coopération - nous reviendrons sur cette idée plus tard) où des agents munis de dispositions ou d'habitus différents luttent (ou coopèrent) pour l'obtention de profits symboliques (ou économiques). Dans l'analyse de l'entreprise de la traduction, le traducteur est l'agent privilégié «par lequel passe la logique objective du système de pratiques, de dispositions et de structures permanentes et générales qui sont à l'œuvre dans toute traduction» (Gouanvic 1999: 17). Mais il n'est pas le seul à intervenir: aux responsables de la traduction, non seulement dans les différentes instances du champ littéraire (éditeurs, directeurs de collection, agents littéraires, critiques), s'ajoutent des agents et des organismes "affiliés» à des institutions supra-littéraires (attachés culturels des ambassades, agents «culturels» des Affaires étrangères et du ministère de la Culture, associations universitaires, etc.) dont, nous le montrerons, l'action peut avoir une incidence directe sur l'entreprise de la traduction, surtout en ce qui concerne l'exportation et la promotion de la littérature traduite dans les espaces culturels cibles.

Malgré la pertinence du modèle bourdieusien en traductologie, il convient de réviser ou plutôt de nuancer certaines idées au cœur de ce modèle, notamment sur le concept de «lutte». Pour Bourdieu, le principe générateur et unificateur du champ est la lutte même, et les différentes prises de position dans un champ donné sont le produit et l'enjeu d'un conflit permanent (Bourdieu 1998). Bourdieu parle presque 
toujours en termes agonistiques et aborde rarement la coopération entre les agents faisant partie du même groupe: il passe de la lutte entre les agents du champ ayant des dispositions ou des habitus différents à la lutte entre les différents groupes, mais n'analyse pas la manière dont les agents d'un même groupe, c'est-à-dire les agents occupant une même position au sein du champ, sont liés entre eux. Même quand il aborde la formation des groupes, il fait référence à une "cohésion négative» observable dans la phase d'accumulation initiale du capital symbolique, mais qui s'affaiblit une fois que le groupe accède à la reconnaissance (et que les profits symboliques ou économiques vont à un petit nombre, sinon à un seul). C'est alors que le groupe se dissout (Bourdieu 1998: 439). Autrement dit, pour Bourdieu, la coopération entre les agents ne serait qu'un état transitoire dans la formation de groupes ${ }^{8}$.

Or, le cas que nous étudierons ici révèle le contraire. En effet, si une littérature minoritaire telle que la littérature québécoise réussit à franchir les frontières linguistiques et culturelles et à se ménager un espace de réception dans les champs culturels espagnol et catalan, c'est précisément grâce à la coopération des agents et des institutions des champs sources et cibles qui participent à toutes les phases du transfert littéraire. Comme nous tenterons de le montrer, il y a derrière chaque chaînon du réseau un agent qui coopère avec celui qui le précède et celui qui le suit.

En fait, comme l'indique Boisclair pour le champ littéraire (2004: 84-85), il y existerait deux axes: sur l'axe vertical, les agents des différents sous-champs sont en concurrence, mais sur l'axe horizontal, les agents sont en situation de coopération. La même chose pourrait s'appliquer aux agents appartenant à des institutions supralittéraires. C'est précisément sur ce dernier axe, "l'axe de la coopération», que nous nous concentrerons pour tenter d'expliquer l'existence et la réception postérieure de la fiction québécoise en Espagne et en Catalogne. Si, au contraire, on essayait d'expliquer l'existence de ce corpus en termes de cohésion négative, c'est-à-dire, en argumentant, par exemple, que la littérature québécoise, ainsi que d'autres littératures dites minoritaires, sont traduites pour contrecarrer le monopole (en Espagne et ailleurs) d'une littérature dominante telle que la littérature états-unienne, on aurait moins affaire à une hypothèse plausible qu'à une théorie du complot anti-américain. Même si l'on acceptait que l'anti-américanisme de certains agents et institutions puisse éventuellement agir comme facteur les motivant à participer au transfert de la littérature québécoise en Espagne, il serait naïf et simpliste de réduire l'existence de cette littérature en Espagne et, surtout en Catalogne, à une seule cause. En effet, les causes incitant les acteurs et les agents institutionnels des champs source et cible à investir ou non dans l'entreprise de la traduction sont multiples. Mais si multiples soient-elles, c'est l'anticipation de profits (économiques pour certains agents, symboliques pour d'autres) qui conciliera les différentes causes à la base des actions des participants et garantira leur coopération (Pym 1998). Nous en montrerons plusieurs exemples.

En résumé, le concept de lutte ne s'avérant pas opératoire, nous avons eu recours à un autre concept, celui de coopération - ou d'«association » pour employer la terminologie de Callon (1986) - et, par extension, à celui de « réseau». Selon Exler : «La disposition à coopérer dépend de l'estimation qu'effectue l'individu du gain qu'il ambitionne et de la perte qu'il subira en cas d'échec» (1999: 2). Pour que la coopération soit durable, il faut inciter les agents à coopérer pour l'obtention d'un gain relatif plutôt que maximal. De plus, pour que la coopération entre les agents soit 
efficace, il faut qu'ils soient organisés d'une manière donnée, à savoir sous forme de «réseaux». Pour Latour, le réseau ${ }^{9}$ associe différents protagonistes, "dotés de modes d'existence et de facultés très hétérogènes » qui - par un processus de "traduction », c'est-à-dire, par une succession d'opérations de délégation, de médiation - «accepteront» (dans le meilleur des cas) que d'autres parlent à leur place. Ces chaînes de porte-parole transportent d'un point à l'autre des entités enrôlées derrière elles (Claisse 2006: 33). Le concept de réseau est donc un descripteur, « « un véhicule» approprié pour «suivre les acteurs» au plus près de leurs déplacements» (Claisse 2006: 33) pour voir comment leur coopération se formalise et se stabilise.

Ici, la volonté de coopérer est à la base de la constitution d'un réseau. Cependant, la notion de coopération chez Latour et Callon n'a rien d'idyllique. Selon eux, la controverse précède toujours l'émergence d'un énoncé scientifique et d'une innovation, et la reconfiguration d'un réseau doit toujours passer par l'analyse des controverses qui entourent la production de connaissances. Après l'analyse de ces controverses, les chercheurs relient des énoncés et des enjeux a priori incommensurables, et rendent le réseau intelligible, sans pour autant simplifier les négociations, les tensions, mais aussi les alliances entre les agents constituant ce réseau.

Cela indiquerait-il que les positions de Bourdieu et de Latour ne sont pas si incompatibles qu'on avait pu le croire, mais plutôt complémentaires? En traductologie, Buzelin (2005) et Inguilleri (2005) ont déjà abordé la potentielle complémentarité des deux théories. Selon la première auteure, cette complémentarité est envisageable: qui plus est, à plusieurs reprises, le modèle de Latour permet de dépasser et de combler les lacunes de celui de Bourdieu. Pour Inghilleri, en revanche, les approches sociologiques en traductologie doivent partir de la position épistémologique de Bourdieu et en cas de points de contingence entre celui-ci et d'autres modèles, notamment avec celui de Latour, le positionnement épistémologique de Bourdieu doit primer. Au fil de cet article, nous laisserons voir que notre position se rapproche plutôt de celle de Buzelin.

Outre la notion de coopération, il faut également s'interroger sur l'utilité de cet explicans et cet explicandum qu'est le réseau ${ }^{10}$, autrement dit, faire état des ajustements épistémologiques auxquels nous avons soumis la notion de réseau, telle qu'elle est conçue par Latour et Callon, pour la rendre apte à l'analyse de la fiction québécoise traduite en Espagne. N'oublions pas non plus que le modèle de Latour et Callon est conçu pour rendre compte de l'activité scientifique et non de l'activité littéraire. D’une part, nous mobilisons la notion de «réseau» dans un sens moins métaphorique que celui du modèle de Latour et Callon ${ }^{11}$ : le réseau représente pour nous un instrument qui sert à décrire des relations effectives, c'est-à-dire empiriquement observables, des agents et des acteurs. En revanche, quand Latour affirme que les chaînes d'acteurs forment un réseau, ce n'est qu'une «façon de parler» (Claisse 2006). Le réseau n'est pas un réseau de relations entre individus, mais une association entre entités hétérogènes (ces dernières pouvant être des humains et des non-humains), qui «acceptent» (emploi métaphorique lorsque l'entité est un non-humain) un porte-parole censé parler en leur nom ${ }^{12}$.

La notion de réseau ainsi conçue et liée à la notion de coopération dont nous avons déjà parlé pourrait servir de complément à la théorie des champs de Bourdieu: une approche bourdieusienne permettrait d'étudier les relations objectives ${ }^{13}$, surtout concurrentielles, entre les agents et les acteurs, tandis que celle des acteurs-réseaux 
se centrerait sur les relations effectives de coopération des agents. Soit, d'un côté, le processus interne d'enrôlement et de mobilisation des réseaux (Callon 1986) - c'està-dire le mécanisme par lequel un rôle est attribué à un acteur ou un agent qui l'accepte, ainsi que l'implication des acteurs ou des agents dans l'action et, donc, la cimentation du réseau - et, de l'autre, l'analyse des rapports (concurrentiels) entre les agents ou les acteurs occupant des positions différentes au sein d'un même champ ou de champs différents (et, par conséquent, appartenant à des réseaux différents). Enfin, si la sociologie du champ permet d'examiner certains des facteurs liés à sa structure à un moment donné, facteurs qui peuvent avoir une incidence directe sur la production et la circulation des traductions, il en va autrement de la sociologie des réseaux, fondée sur la prémisse selon laquelle pour expliquer les interactions entre les acteurs nul n'est besoin de recourir à l'existence de facteurs structurels. Dès lors, dans notre étude, le réseau apparaîtra non comme une méthodologie à part entière (Sapiro 2006: 46), mais comme un concept opératoire, «une méthode [servant] à explorer le champ littéraire». De même, pour Lacroix, la théorie des champs «ne possède pas un pouvoir omni-explicatif et ne peut pas rendre compte de tout ce qui est social dans la vie littéraire; or, un de ces aspects qu'[elle] ne saisit qu'imparfaitement est celui des relations concrètes, dont l'analyse de réseau offre, elle, une analyse stimulante» (Lacroix 2006: 202).

D’autre part, il convient de nuancer la notion d'hétérogénéité, qui occupe une place de choix dans la théorie des acteurs-réseaux. L'étude de cas que nous présentons insiste également sur le caractère hétérogène de la notion de réseau, mais non en tant que combinaison d'acteurs humains et non humains, telle que la conçoit Latour: cette notion est mobilisée pour rendre compte des interactions d'agents qui appartiennent à des milieux divers. En effet, sera révélée l'existence d'un macro-réseau composé aussi bien de réseaux institutionnels et semi-institutionnels (très structurés et formels où le rôle des agents est normalement interchangeable et bien défini) que de réseaux proprement littéraires (plus flous et informels, aux interconnexions relativement aléatoires et dont la continuité n'est jamais garantie). Nous verrons que l'action des premiers, soit les réseaux les plus institutionnalisés, est cruciale car ce sont eux qui activent les réseaux littéraires. Autrement dit, nous montrerons que dans le cadre d'un champ littéraire relativement jeune tel que le champ québécois, même l'activation des réseaux littéraires les plus flous et informels passe habituellement par la médiation d'un agent ou d'un acteur affilié à un réseau institutionnel, qui fonctionne lui aussi selon une logique de réseaux. Cela pourrait indiquer que dans les champs littéraires dominés ou périphériques, où la négociation est constante entre les logiques littéraire et politique, les réseaux littéraires et les réseaux extra-littéraires sont étroitement interconnectés.

En d'autres termes, notre étude montrera que les réseaux internationaux littéraires qui s'établissent entre les écrivains ou les éditeurs des champs littéraires sources et cibles passent presque toujours par un intermédiaire qui fait partie des réseaux institutionnels. Selon Sapiro: «L'analyse des relations entre les divers types de réseaux [...], des plus institutionnalisés aux plus informels, permettrait de repérer la multipositionnalité et le rôle d'intermédiaire parfois occulte de certains individus » (2006: 56). Cela veut dire que, comparativement aux réseaux d'autres champs littéraires dominants qui sont activés directement par des connexions entre des agents du champ littéraire proprement dit, dans le cas de la fiction québécoise, intervient fréquemment 
un intermédiaire institutionnel. Une fois cette mission accomplie, c'est aux agents littéraires de maintenir et de consolider leurs réseaux. À ce travail d'impulsion et d'activation des réseaux institutionnels, succède le chaos et l'imprévisibilité, imprévisibilité que la théorie des champs ne permet pas d'aborder.

\section{Réseaux en action et action des réseaux : la fiction québécoise traduite en Espagne}

Sur ces bases théoriques, nous examinerons maintenant la formation et le fonctionnement interne, le sens et les intérêts investis des réseaux institutionnels qui, parallèlement à d'autres agents et acteurs du champ littéraire, rendent possible l'existence d'un espace de réception pour la fiction québécoise en Espagne et en Catalogne.

À partir d'entrevues semi-structurées ${ }^{14}$ menées avec des agents-clés choisis en raison de leur intermédiarité ${ }^{15}$ (Degenne et Forsé 1994: 154, cités par Lacroix 2003), ainsi que de données complémentaires obtenues par d'autres moyens (notamment, grâce à la correspondance échangée avec des agents-clés, à des références croisées dans les publications de certains agents, aux programmes d'événements culturels ou universitaires reconnus comme des lieux de sociabilité clés pour le développement de ces réseaux), nous avons pu tracer une cartographie où trois types de réseaux interviennent et interagissent: les réseaux officiels (c'est-à-dire publics, ou étatiques), les réseaux associatifs universitaires et les réseaux proprement littéraires.

\section{a) Les réseaux officiels}

Le Canada et l'Espagne sont des pays qui partagent des problématiques communes: une structure politique décentralisée, des nationalismes internes très actifs et des défis propres à l'intégration de leurs immigrants. Ces similitudes ont sans doute rapproché les deux pays, de même que des interactions privilégiées entre certains de leurs territoires, le cas le plus notoire étant celui des relations qu'entretiennent la Catalogne et le Québec.

Sur le plan des États, les relations entre le Canada et l'Espagne, bien que relativement récentes (le premier accord en matière de culture date de 1989), sont de plus en plus dynamiques. Les relations du Québec avec l'Espagne sont également récentes, mais elles connaissent une croissance exponentielle ${ }^{16}$ et se concentrent - principalement, mais non exclusivement - en Catalogne. Le dynamisme et l'intensité des rapports entre la Catalogne et le Québec sont tels qu'ils signent en 1996 une entente-cadre de coopération sur plusieurs plans, y compris culturel, la seule entente-cadre entre le Canada et l'Espagne. La quête identitaire commune de ces deux provinces, autant sur le plan culturel que politique, constitue certainement un point de rapprochement.

Bien que ces relations ne soient pas sans effet sur la manière dont l'Espagne ou la Catalogne abordent les productions littéraires venant du Canada, elles ne suffiront pas. Pour que ces productions existent et pour leur fournir un espace de circulation en Espagne, plusieurs réseaux sont mobilisés par les gouvernements fédéral canadien et provincial québécois suivant un processus d'«exoformation»(Gellner 1989), à savoir un processus d'institution d'une culture par des institutions spécialisées en collaboration avec des associations semi-privées et des individus provenant autant des espaces sources que cibles. 
Les réseaux officiels, sur les plans fédéral et provincial, fonctionnent de manière relativement autonome. À l'intérieur de chaque réseau, il convient de distinguer les acteurs représentant le Canada et le Québec en Espagne in situ, et ceux qui agissent à partir des espaces sources et se servent parfois des premiers comme intermédiaires. Sur le plan fédéral, deux acteurs principaux participent au transfert de la littérature québécoise en Espagne et en Catalogne: l'Ambassade du Canada en Espagne et le Conseil des Arts du Canada. Liés à ces derniers, nous pouvons repérer deux réseaux associatifs: l'Association pour l'exportation du livre canadien (l'AELC), et l'autre de caractère universitaire, soit l'Asociación Española de Estudios Canadienses (l'AEEC). L'Ambassade du Canada en Espagne dépend directement du ministère des Affaires étrangères du Canada (MAÉCI) et représente le Canada (dont le Québec) sur l'ensemble du territoire espagnol. Cette ambassade compte une section culturelle qui veille à la connaissance du Canada et de ses expressions culturelles sur l'ensemble du territoire espagnol. Cette section culturelle de l'Ambassade reçoit divers fonds (directs, indirects, réguliers et sporadiques) provenant de différents ministères du gouvernement fédéral du Canada (Laura Ballesteros, en entretien en 2006). Elle coopère donc de manière différente (selon le type de fonds) avec les ministères et d'autres acteurs (p. ex. le Conseil des Arts) qui lui fournissent ces fonds.

Les plus intéressants pour notre étude sont les fonds directs, c'est-à-dire directement gérés par l'Ambassade et provenant du MAÉCI, de son programme de diplomatie publique - la somme totale pour 2006-2007 s'élevant à 10000 \$ (Ballesteros 2006) -, ainsi que les fonds indirects additionnels reçus du MAÉCI et du Conseil des Arts du Canada. Commençons par le premier type. La diplomatie publique - aussi dénommée «diplomatie douce», «diplomatie ouverte» ou «diplomatie culturelle» - est le troisième pilier de la politique étrangère canadienne (1995) et comprend une série d'initiatives menées par le MAÉCI, surtout à partir des années 90, dont le but est de promouvoir l'identité et les valeurs canadiennes à l'étranger et de renforcer l'internationalisation du Canada. Encore aujourd'hui, dans l'Énoncé de politique internationale du Canada (2005), la diplomatie publique demeure une priorité. Potter la définit comme " [...] the effort of the government of one nation to influence public or elite opinion of another nation for the purpose of turning the policy of the target nation to advantage» (2002: 3). En vertu de cette nouvelle forme de diplomatie, la réputation et l'influence du Canada à l'étranger ne reposent pas seulement sur l'action gouvernementale, mais aussi sur les citoyens canadiens (artistes, enseignants, étudiants, voyageurs, chercheurs, etc.) qui entreront en contact avec d'autres agents et donc avec d'autres réseaux internationaux à l'étranger: «The government will become more of a facilitator and less of a doer» (Potter 1996: 6). En d'autres termes, le rôle de ce réseau officiel fédéral est de faciliter l'activation d'autres réseaux informels (en l'occurrence, littéraires) qui, une fois activés, seront hors du «contrôle» des premiers : par exemple, seront encouragées, organisées et subventionnées des rencontres d'écrivains, d'éditeurs, etc., des aires sources et cibles, pour qu'ultérieurement ces derniers établissent leurs propres réseaux, des réseaux aux frontières plus poreuses et aux conditions d'accès moins codifiées. Ces réseaux, plus flous, seront basés sur les affinités des agents en faisant partie, plutôt que sur des rapports imposés d'en haut. Mais quel est l'intérêt du gouvernement fédéral, et quels sont les objectifs qui orientent ce travail d'activation de réseaux sur lesquels il n'aura aucun pouvoir une fois ces derniers activés? 
Les motivations sous-jacentes à la mise en place de ces initiatives de diplomatie publique semblent présenter une double nature, économique et identitaire. Pour encourager les entreprises étrangères à investir au Canada, il fallait créer une nouvelle «image de marque»; dans les années 90, le Canada était encore perçu comme un pays basé sur une économie de ressources, comme au siècle passé (Potter 2004). Il fallait donc le (re)présenter comme un pays dynamique, innovateur, doté d'une technologie avancée, mais également tolérant, compétitif, bilingue et multiculturel (Potter 2002). La diplomatie culturelle a déployé de multiples outils à cet effet: événements culturels, conférences, bourses pour des étudiants étrangers au Canada, implantation d'associations d'études canadiennes à l'étranger, etc. C'est au cœur de ce projet que se situe la traduction d'œuvres canadiennes. La traduction des produits culturels canadiens (non seulement des livres, mais d'autres formes artistiques comme, par exemple, le cinéma) constitue un outil très important au service de la diplomatie publique canadienne. De fait, selon John Ralston Saul (1994): «Many foreigners, when they think of Canada, think first of these writers and books [Canadian writers that have won large publics outside of Canada]. This is what they know». Malgré tout, la traduction est rarement mentionnée dans le discours officiel ou universitaire sur la diplomatie publique (Oeding et von Flotow 2005).

La diplomatie publique répond également à des motivations identitaires (GutiérrezHaces 2003): tout en encourageant une certaine identité et des valeurs dites canadiennes à l'étranger, elle permet de renforcer «l'identité nationale canadienne» à l'intérieur du Canada. Comme le dit Potter: "The absence of a vibrant, sustained and coordinated presence abroad through culture and education stating, "This is Canada", could prompt a disturbing question, "What is Canada?" " (2002: 18) et ce, même au Canada. La traduction de la littérature canadienne constituerait donc aussi un mécanisme de réaffirmation identitaire canadienne - ou plus précisément un mécanisme de renforcement du récit officiel fédéral sur l'identité canadienne - à l'étranger et, indirectement, au Canada.

Que dire alors de la traduction de la littérature québécoise en particulier? Les initiatives et l'appui financier du gouvernement fédéral à cet égard pourraient être qualifiés de stratégiques par rapport au fait francophone, l'élément canadien-français servant à marquer la singularité d'une Amérique française au Canada. Ceci permet, dès lors, de distinguer le Canada (et plus précisément le Canada anglais) des ÉtatsUnis. Même si, dans le cadre des politiques étrangères canadiennes de 1995 et de 2005, le bilinguisme perd son rôle principal au profit du multiculturalisme, la mobilisation du fait francophone semble bel et bien être une constante dans le cadre de ce récit officiel sur le Canada ${ }^{17}$.

Ainsi, les fonds directs que reçoit la section culturelle de l'Ambassade du Canada en Espagne dans le cadre du programme de diplomatie publique répondraient aussi aux motivations que nous venons d'exposer. Ils sont gérés directement par l'agente d'éducation et de culture, Laura Ballesteros, et sont destinés à des activités très diverses, et ce, même si le rôle de l'Ambassade est réactif plutôt que proactif (Ballesteros 2006). L'Ambassade finance en partie ou collabore à l'organisation d'événements culturels au cours desquels le Canada est représenté, tels que PhotoEspaña (photographie), ARCO (art contemporain), le festival annuel du cinéma canadien ou la foire du livre de Liber; elle peut également soutenir des activités promotionnelles ou des rencontres entre des écrivains canadiens et des journalistes espagnols, pro- 
duire des invitations et affiches arborant le logo de l'Ambassade (pour tout genre d'événement culturel lié au Canada), appuyer des activités universitaires à contenu canadien organisées par l'Asociación Española de Estudios Canadienses (AEEC). Laura Ballesteros joue un rôle pivot entre l'Ambassade et une association savante telle que l'AEEC, qui compte parmi ses réseaux des membres faisant partie d'autres réseaux des espaces cibles, en l'occurrence des réseaux littéraires (par exemple des directeurs de collection des maisons éditoriales). Ils constituent donc des nœuds importants qui connectent des réseaux universitaires liés à la littérature canadienne et des maisons d'édition susceptibles de publier cette littérature. Par exemple, Landry-Wilfrid Miampika, professeur à l'Université d'Alcalá, spécialiste en littérature africaine et des Antilles, est en relation directe avec des membres du réseau de l'AEEC $^{18}$, comme le montrent les références croisées de ses publications et la liste des communications qu'il a présentées. Il est directeur de la collection «La Diversidad» (série francophone) et c'est lui qui a suggéré à l'éditrice de Ediciones el Cobre, Miriam Tey, de traduire et de publier Esta granada en manos del joven negro ¿es un arma o una fruta? (2004) de Dany Laferrière (Communication personnelle avec Marta Tey, de Ediciones El Cobre, le 26 janvier 2007). Voilà un exemple de réseau formé et constitué par des agents aussi bien institutionnels que plus proprement littéraires.

En matière d'édition, en plus des activités de promotion déjà mentionnées, l'Ambassade travaille depuis 2002 avec l'Association pour l'exportation du livre canadien (l'AELC) afin de rapprocher les maisons d'édition espagnoles (dont les catalanes) et canadiennes (dont les québécoises) (Ballesteros 2006). Par exemple, l'Ambassade ${ }^{19}$ a encouragé et organisé la participation de l'AELC à la foire du livre LIBER, qui a lieu à Madrid ou à Barcelone, où des éditeurs canadiens et québécois ont été présents en 2004 et 2005, et seront représentés en 2007. C'est le début d'un rapprochement présentement en cours, dont les résultats n'ont pas encore été évalués (Ballesteros 2006). Ici encore, le rôle de l'Ambassade est d'intervenir en tant qu'intermédiaire pour activer des liens entre les éditeurs des espaces culturels sources et cibles, mais ce sont les éditeurs de chaque côté qui doivent travailler dans leurs champs respectifs à la continuité de ce rapprochement et à la consolidation des réseaux internationaux activés grâce à ces rencontres organisées par Laura Ballesteros.

Quoi qu'il en soit, la présence progressive de livres canadiens sur le marché espagnol et catalan semble indiquer un lien de plus en plus étroit entre les industries éditoriales des espaces sources et cibles ${ }^{20}$. Si l'on observe les dates figurant au tableau des traductions de la fiction québécoise présenté en annexe, on note quavant les années 90, la moyenne est de 1,1 livre par an, tandis qu'à partir de cette date, la moyenne est de 4,1, avec des années comme 2003 atteignant les 9 titres publiés. Pour le livre canadien en général, genre et langue confondus, une progression y est également observable ${ }^{21}$. En ce qui concerne les transferts Canada-Espagne, l'édition constitue donc une industrie «en voie de développement» certes prometteuse, mais elle est à l'heure actuelle précédée (par ordre d'importance) par les arts visuels, audiovisuels et scéniques (Ballesteros 2006), lesquels ont plus de retombées en Espagne.

L'Ambassade reçoit également deux types de fonds indirects intéressants à considérer ici: le programme Promart (Promotion des arts) du MAÉCI, qui fournit une aide financière pour des projets promotionnels, y compris des tournées de lectures et d'événements médiatiques visant à stimuler l'intérêt autour des œuvres littéraires canadiennes à l'étranger, ainsi que pour la participation d'écrivains à d'importantes 
foires internationales du livre. Les demandes sont approuvées à Ottawa, mais Laura Ballesteros peut soumettre des recommandations sur les projets à subventionner avant qu'une décision finale ne soit prise. Cela signifie qu'il existe un réseau institutionnel fédéral, avec des agents-clés à Ottawa et à Madrid, qui connaissent bien les contextes sources et cibles, et coopèrent afin d'appliquer le programme de diplomatie publique au contexte espagnol et d'en assurer le bon déroulement.

Enfin, pour compléter la composition de ce réseau officiel fédéral, il faut ajouter un autre acteur, le Conseil des Arts du Canada, qui, en partenariat avec le MAÉCI jusqu'en 2005 (date à laquelle ce partenariat a pris fin, sans que le Conseil ne cesse pour autant d'attribuer des subventions), offre aussi des subventions à la traduction internationale d'œuvres canadiennes, ainsi qu'à la promotion de ces traductions. Le Conseil des Arts est un acteur différent; en effet, bien que la totalité des fonds qu'il reçoit provienne du gouvernement fédéral (en particulier du ministère du Patrimoine canadien, et pour des programmes ponctuels du MAÉCI), il s'agit d'un organisme national et autonome qui, en vertu du principe d'autonomie, dispose d'une complète indépendance à l'égard du gouvernement ${ }^{22}$. En pratique, il est difficile de croire que l'orientation des politiques et les programmes du Conseil des Arts ne fassent pas écho, du moins en partie, à celles du gouvernement fédéral. Cette question, cependant, est quelque peu polémique et serait l'objet d'un autre article.

Après les Pays-Bas, l'Italie et l'Allemagne, l'Espagne se situe parmi les pays qui ont reçu le plus de subventions du Conseil, pour un total s'élevant à 76. Pour le cas particulier de la fiction québécoise, 20 traductions du corpus présenté en annexe ont été subventionnées ( $13^{23}$ pour la jeunesse et $7^{24}$ pour les adultes). Quant aux critères à partir desquels le Conseil décide d'accorder ou non des subventions, à la fiabilité de l'éditeur étranger concerné s'ajoutent d'autres critères liés à l'original: par exemple, le fait que celui-ci ait gagné ou ait été en lice pour les Prix du Gouverneur général. D’ailleurs, comme Montgomery (2006) le souligne, il s'agit également d'une façon de faire la promotion de ces prix.

Le choix des traductions espagnoles et catalanes subventionnées par le Conseil semble répondre en général aux priorités stratégiques de ce dernier, à savoir la littérature pour la jeunesse et la littérature migrante (Montgomery, en entretien, 2006). Cependant, d'autres «traits» sont repérables dans ces traductions subventionnées, par exemple l'internationalisation du Canada, c'est-à-dire l'importance accordée à sa projection internationale, comme c'est le cas dans Un diumenge a la piscina a Kigali. De même, on est à l'écoute d'une voix de femme dans El soroll de les coses vives, et aussi de la spécificité québécoise, ou plus précisément, de la francophonie américaine, notamment dans La nena que li agradaven massa els llumins et Gatuperios, œuvres où non seulement la scène se situe au Québec (un Québec rural pour la première et urbain pour la deuxième), mais où les québécismes pullulent. Bref, dans l'échantillon limité d'œuvres francophones subventionnées, sont représentés la jeunesse, les émigrants, les femmes et la spécificité québécoise, en tant que spécificité francophone des Amériques, qui, curieusement, constituent autant de traits au cœur de «l'image de marque» du Canada que le MAÉCI souhaite projeter à l'étranger. Serait-ce que les éditeurs (ce sont eux qui doivent déposer une demande de subvention) ne s'intéressent tout simplement pas à des aspects autres que ces traits, ou que le travail d'élaboration d'une image de marque du Canada mené du point de vue institutionnel commence à porter fruit? 
L'approbation de ces subventions relève exclusivement du Conseil des Arts, ce qui sous-entend que ni le MAÉCI ni l'Ambassade n'ont de pouvoir de décision dans la sélection des traductions à subventionner (Montgomery 2006). Il arrive cependant que le Conseil des Arts consulte Laura Ballesteros sur la réputation des maisons d'édition espagnoles ayant déposé une demande (Montgomery 2006).

En ce qui concerne la traduction de la littérature québécoise en Espagne, s'ajoute au réseau institutionnel fédéral un autre réseau provincial québécois, qui est composé de plusieurs acteurs, dont le Bureau du Québec à Barcelone (désormais «BQ Barcelone»), qui joue un rôle central dans le cadre de l'entreprise de la traduction de la littérature québécoise en Espagne, et d'acteurs très secondaires pour notre étude qui complètent le rôle du premier, soit la Société de développement des entreprises culturelles du gouvernement du Québec (SODEC) et le Conseil des arts et des lettres du Québec (CALQ).

Le BQ Barcelone représente officiellement le Québec en Espagne. Bien qu'au départ il ait été créé (en 1999) pour répondre à un mandat exclusivement commercial - et non politique ${ }^{25}$ - après que l'on se fut rendu compte que «la meilleure forme de diplomatie [commerciale] est la culture» (Bronchal 2006), on a élargi son mandat initial. À l'heure actuelle, le secteur culturel, sous la direction de María Jesús Bronchal Torres, attachée aux affaires publiques et culturelles (un autre agent-clé de notre cartographie), constitue un domaine prioritaire. Dans le cadre de son mandat culturel, le BQ Barcelone assure la promotion et la diffusion de la spécificité culturelle québécoise dans l'ensemble de l'Espagne, tout en entretenant avec la Catalogne «une relation privilégiée, intouchable» (Bronchal 2006) en vertu de l'entente-cadre entre le Québec et la Catalogne signée en 1996. Une des priorités du BQ Barcelone est donc de mettre en œuvre et de faire le suivi de l'accord de coopération entre le Québec et la Catalogne: le secteur de l'édition - et, partant, la traduction - font partie de cette coopération.

La section culturelle du BQ reçoit aussi des fonds directs et indirects des ministères avec lesquels elle coopère (Bronchal 2006): des fonds directs provenant du ministère des Relations internationales et destinés exclusivement à l'accord de coopération Québec-Catalogne, et des fonds du ministère de la Culture et des Communications du Québec pour des activités culturelles réalisées sur l'ensemble du territoire espagnol, avec une tendance à exclure la Catalogne (Bronchal 2006), qui est déjà couverte par l'accord de coopération. Cependant, de l'avis de cette dernière, l'accord de coopération avec la Catalogne est prioritaire, des priorités régionales et sectorielles ayant été définies.

Des fonds indirects provenant des autres acteurs avec lesquels le BQ collabore, soit l'Association internationale des études québécoises (AIÉQ), la Société de développement des entreprises culturelles du gouvernement du Québec (SODEC) et le Conseil des arts et des lettres du Québec (CALQ), sont également disponibles pour le financement de tournées d'artistes québécois en Espagne. Ces fonds sont destinés à des activités très variées - principalement, mais non exclusivement - concentrées en Catalogne: rencontres de poètes, programmes de résidences d'artistes en Catalogne et au Québec, organisation, en collaboration avec l'ANEL, de la participation des éditeurs québécois à des salons ou des foires du livre, identification d'agents-clés et invitations à visiter le Québec ou les salons du livre où le Québec est représenté (comme la foire de Guadalajara au Mexique). Il faut remarquer que les salons et les 
foires sont des lieux primordiaux de rencontre et de sociabilité pour les éditeurs. De plus, ces rencontres ne sont pas fortuites, mais bel et bien planifiées (Bronchal 2006), et elles permettent de former et de consolider les réseaux entre les éditeurs des différentes aires culturelles. Elles sont également financées par les gouvernements fédéral et provincial. Et ce, non seulement pour aider les éditeurs, mais aussi parce qu'on sait qu'il s'agit d'endroits stratégiques pour promouvoir le Canada ou le Québec à l'étranger.

Ici encore, il s'agit d'activer des réseaux en dehors de la sphère institutionnelle, lesquels acquerront (ou non) une vie propre. En ce sens, pour montrer le genre de travail que réalise le BQ Barcelone et les résultats éventuels, María Jesús Bronchal (2006) cite l'exemple de l'invitation que le BQ a faite à Segimon Borrás (secrétaire général de l'Association des écrivains en langue catalane et organisateur, en collaboration avec d'autres, de la foire internationale du livre LIBER à Barcelone) de visiter le Québec et le fait que - même si ce n'est pas encore confirmé - le Québec sera en 2008 le «pays» invité de cette foire.

En somme, comme la section culturelle de l'Ambassade (mais avec des fonds plus modestes), le BQ Barcelone se livre à un important travail d'impulsion initiale pour que les parties (dans n'importe quel secteur) puissent par la suite activer des réseaux et les consolider sans appui institutionnel. Une fois la collaboration implantée, c'est le BQ qui «tire profit» des écrivains québécois (Bronchal 2006) en se servant des contacts qu'ils ont établis dans l'espace littéraire cible. María Jesús Bronchal soulève notamment le cas de Nicole Brossard. En fait, celui-ci est intéressant parce que grâce à ces programmes de résidences de poètes en Catalogne et au Québec organisés par le BQ Barcelone en collaboration avec l'Institució de les Lletres Catalanes, elle a pu se faire connaître auprès du réseau de poètes catalans avec lesquels elle a maintenu des relations; c'est d'ailleurs le poète catalan Pere Gimferrer qui a influencé la décision de traduire Baroque d'aube à la maison Seix Barral, traduction qui a été publiée en 1998.

En matière d'édition et particulièrement de traduction, et en ce qui concerne le genre romanesque, le BQ a mis sur pied un projet de collaboration entre la SODEC et l'Institució de les Lletres Catalanes pour la traduction d'œuvres québécoises vers le catalan, et inversement. Il s'agissait d'élaborer une liste des œuvres à traduire et de la soumettre aux maisons d'édition, lesquelles obtiendraient des subventions à cet effet. Le projet, cependant, n'a pas abouti en raison de la réponse négative des éditeurs catalans (la contrepartie québécoise avait, en revanche, répondu par l'affirmative), car comme le souligne María Jesús Bronchal (2006): "La mentalité des maisons d'édition en Catalogne ne permet pas qu'on leur impose des titres, pas plus qu'elles ne souhaitent s'en faire suggérer. Elles refusent que «l'Institution» leur impose une façon de faire». Cela montre aussi qu'une participation directe des acteurs et des agents institutionnels dans des réseaux éditoriaux plus proprement littéraires n'est pas une démarche fructueuse, mais que la planification institutionnelle doit se centrer sur la création des conditions favorables pour que les agents littéraires de différents champs s'allient entre eux.

Malgré des échecs ponctuels dans des projets comme celui que nous venons de mentionner, toutes les activités visant la promotion de la littérature québécoise, avant et après la traduction des œuvres, contribuent non seulement à encourager la traduction d'œuvres québécoises auprès des éditeurs catalans, mais aussi à leur préparer un 
accueil favorable dans l'aire culturelle catalane. On observe donc que sur le plan provincial, ce qui est planifié et géré par l'action du BQ - et son réseau d'agents et d'acteurs - a peu à faire avec le transfert en tant que tel (notons qu'aucune traduction espagnole ou catalane de fiction québécoise n’a été subventionnée par le gouvernement provincial québécois), mais touche plutôt à la promotion.

Celle-ci, cependant, ne suffit pas: bien que le rôle des réseaux institutionnels puisse largement contribuer à l'initiation de ces traductions, et, dans une phase ultérieure, influencer leur réception critique, pour que cette littérature capte l'attention de l'institution littéraire, il doit y avoir un certain intérêt au sein de la communauté réceptrice envers la communauté source. Comme l'indique María Jesús Bronchal (2006): «Il est question de séduire, et non d'imposer. N'importe quelle collaboration imposée d'en haut ne fonctionne pas. Elle ne fonctionne qu'une fois, parce qu'elle n'est pas issue d'un intérêt partagé. Il n'y a pas de continuité».

Dans ce cas-là, il est évident que la Catalogne - en raison des affinités idéologiques qu'elle partage avec le Québec - fait preuve d'un grand intérêt pour tout ce qui est québécois. Mais ne soyons pas naïfs par rapport à cet intérêt: en célébrant le nationalisme d'une autre nation comme le Québec, la Catalogne célèbre en quelque sorte son propre nationalisme. En même temps, le Québec a intérêt à ce que sa littérature et son identité soient projetées à l'étranger, comme on peut l'observer dans le cadre de sa politique internationale. Il s'agit donc clairement d'un double exercice de diplomatie dont profitent les deux communautés, source et cible. Une autre preuve de cet intérêt réciproque résiderait dans le fait que les institutions de l'espace cible subventionnent aussi la traduction de la fiction québécoise en Catalogne. Dans le domaine culturel notamment, l'Institució de les Lletres Catalanes a appuyé, conjointement avec le BQ, des rencontres entre des écrivains québécois et catalans, ainsi que la traduction d'anthologies de poésie québécoise; elle a également subventionné deux traductions catalanes d'œuvres québécoises ${ }^{26}$ : Un dimanche à la piscine à Kigali de Gil Courtemanche (traduction qui a été subventionnée par le Conseil des Arts du Canada) et Putain de Nelly Arcan. En revanche, aucune traduction espagnole ou catalane de la fiction québécoise n'a reçu d'aide financière provenant du gouvernement central espagnol. En d'autres termes, le réseau provincial québécois se lie au réseau catalan pour former un plus grand réseau et ainsi garantir la continuité de ce dernier et, par extension, la continuité de l'entreprise de la traduction de la littérature québécoise en Catalogne.

Néanmoins, les rapports Catalogne-Québec et, par extension, les transferts littéraires entre ces deux espaces diffèrent de ceux qui existent entre le Canada et l'Espagne $^{27}$. Dans ce dernier cas, il ne s'agit pas tout à fait d'un double exercice de diplomatie dans le cadre duquel les communautés source et cible ont investi les mêmes intérêts. Au contraire, c'est plutôt le pôle source, en particulier le gouvernement fédéral canadien et le réseau qu'il déploie, qui se charge d'initier et de promouvoir la traduction de la littérature canadienne (dont la québécoise) sans qu'il existe forcément un intérêt «naturel» préalable dans l'espace cible espagnol, comme c'était le cas pour l'espace catalan. Or, même si l'élan et l'action issus du pôle source - ou des agents du pôle cible, mais étroitement liés à l'espace source, comme les canadianistes et les québécistes espagnols - peuvent certainement initier et promouvoir cette entreprise de traduction, voire influencer la réception de ces traductions par les instances critiques du pôle cible, rien ne garantit ni sa continuité ni une réception 
critique favorable (ou une réception tout court): pour que les traductions soient «consommées», il faut que la communauté cible leur porte un intérêt quelconque.

À cet égard, le cas des traductions espagnoles de l'«écriture migrante» québécoise offre un bon exemple: bien qu'en général la fiction québécoise n’ait pas beaucoup retenu l'attention de la presse culturelle espagnole, les auteurs « ethniques ${ }^{28}$ » - comme les dénomme la presse espagnole - constituent une exception. En fait, cette réception critique favorable pourrait se lire à la lumière de la portée du phénomène de l'immigration en Espagne depuis les années 90: en peu de temps, en effet, le statut de l'Espagne est passé de pays d'émigration à celui de pays d'immigration (AndrésSuárez 2004). À cet égard, à propos de Cómo hacer el amor con un negro sin cansarse (par Dany Laferrière) et de la problématique du racisme et des rapports de pouvoir entre les différents groupes ethniques, María Asunción Guardia, l'auteure du compte rendu sur ce livre paru dans La Vanguardia ${ }^{29}$, écrira: "El libro fue una auténtica revolución en Canadá y [Laferrière] piensa que puede ser útil aquí, ahora que empezamos a convivir con el problema.» [Le livre a entraîné une véritable révolution au Canada et (Laferrière) pense qu'il peut être très utile ici, maintenant que nous commençons à vivre avec ce problème]. Parallèlement à ce commentaire, Inmaculada de la Fuente, auteure d'un compte rendu dans El País ${ }^{30}$, affirme que «Había cierto hastío de historias occidentales y una inevitable saturación de escenarios reconocibles. Era el momento de que llegaran nuevas voces de India, de China, del Pacifico, del Caribe, de África. » [Nous étions las des histoires occidentales, sans compter l'inévitable saturation des scènes reconnaissables. Il était temps que des voix provenant de l'Inde, de Chine, du Pacifique, des Caraïbes et d'Afrique arrivent]. Or, si ces voix parviennent jusqu'à ce pays, c'est grâce à la traduction. Néanmoins, il va de soi que cet accueil critique favorable ne se traduit pas nécessairement par un grand chiffre de ventes. En effet, les éditeurs espagnols qui ont publié des traductions de l'écriture migrante québécoise ${ }^{31}$ et qui ont répondu à nos questionnaires soulignent l'échec commercial de ces traductions ${ }^{32}$.

Comme l'affirment Andrés-Suárez (2004) et Mata (2004), en Espagne, même si la littérature sur l'immigration est relativement abondante, la littérature venant de l'immigration ne l'est pas. L'état du sous-champ de l'écriture migrante en Espagne et en Catalogne est très embryonnaire. À cet égard, on peut considérer qu'à court terme la traduction ${ }^{33}$ vient combler une lacune existant dans les champs littéraires espagnol et catalan où ces voix ne commencent qu'à faire irruption et n'ont pas encore été légitimées par les instances critiques de ces deux champs littéraires. À long terme, la traduction pourrait fort probablement offrir des modèles contribuant à l'émergence et au développement d'une écriture émigrante en espagnol et en catalan. En même temps, elle pourrait préparer le terrain pour que les agents légitimateurs des champs littéraires espagnol et catalan apprennent à écouter et à apprécier la polyphonie florissante des voix littéraires venues d'ailleurs. De même, cet intérêt récent pour la littérature migrante dans le champ littéraire espagnol doit être associé à la relecture actuelle de l'histoire d'Espagne dans les milieux intellectuels. Avec l'arrivée en grand nombre d'immigrants, les Espagnols se disent: «Nous sommes tous des métis », d'où la «relecture de l'œuvre des écrivains migrants-exilés des années 1970 » (Mata 2004: 94). Ici, ce n'est pas seulement l'action des réseaux institutionnels, mais surtout les facteurs structuraux liés à l'état des champs espagnol et catalan qui ont facilité la traduction, ainsi qu'une réception favorable dans les champs littéraires cibles. 


\section{b) Réseaux associatifs}

Parallèlement à ces réseaux officiels et, en quelque sorte, continuateurs de leur mission sur le plan universitaire, figurent deux réseaux de québécistes et de canadianistes institués en associations «savantes». Il s'agit (par ordre d'importance en ce qui a trait à la diffusion de la littérature québécoise en Espagne ou en Catalogne) de l'Association internationale des études québécoises (AIÉQ), association centralisée, mais représentée en Espagne par la professeure Carmen Mata, puis de l'Asociación Española de Estudios Canadienses (AEEC), branche nationale du Conseil international d'études canadiennes, qui vise la promotion des études canadiennes, dont les études québécoises, dans l'ensemble du pays. Ces deux associations semi-privées sont respectivement financées à la fois par les gouvernements provincial pour l'AIÉQ ${ }^{34}$ et fédéral pour l'AEEC ${ }^{35}$, et par les cotisations de leurs membres et les dons versés par des entreprises ou des particuliers. Elles fonctionnent selon une logique de réseaux, toutefois moins institutionnalisés et donc plus flous que les réseaux officiels dont nous venons de parler. Les intermédiaires ou agents-clés de ces réseaux associatifs (par exemple, la professeure Carmen Mata dans le cas de l'AIÉQ) ne représentent pas l'institution pour laquelle ils travaillent; leur adhésion à ces réseaux reflète leurs propres intérêts et motivations. Autrement dit, il s'agit d'un type d'agents qui non seulement participent aux réseaux et en activent d'autres, mais qui en font partie.

Ces deux associations organisent diverses activités (conférences, séminaires, colloques, etc.) et offrent aux québécistes et aux canadianistes espagnols des programmes de bourses pour la recherche, d'aide à la publication d'articles et de monographies, ainsi que du soutien pour la participation à des conférences et pour l'organisation de cours portant sur le Québec et le Canada dans les universités espagnoles et catalanes. En plus de ces subventions, il faut souligner dans le cadre de l'AIÉQ (en collaboration avec le BQ Barcelone, et parfois en collaboration avec l'AEEC), le programme de tournées d'écrivains québécois, qui existe depuis 2002. Ces tournées se déroulent dans des universités à l'étranger et dans des endroits publics, comme les bibliothèques, les librairies, les centres culturels, les instituts français, etc. Dans le cas de l'Espagne, des écrivaines québécoises de notre corpus telles que Marie-Célie Agnant, Nicole Brossard ou Ying Chen ont fait partie de ce programme de tournées. Grâce à celui-ci, des rencontres subséquentes ont lieu entre les écrivains en tournée et les médias locaux, ce qui a une incidence directe sur la promotion de la littérature québécoise en Espagne (les journaux espagnols et catalans publient des entretiens avec les écrivains en tournée, d'où la visibilité accrue de la littérature québécoise dans la presse espagnole, mais surtout catalane). Le but de ces tournées est également d'aller au-delà du monde universitaire et d'organiser des activités dans des bibliothèques et des centres culturels, plus susceptibles d'attirer le grand public que les rencontres universitaires.

Si l'AIÉQ se concentre exclusivement sur les études québécoises et leur diffusion à l'étranger, dont l'Espagne, le mandat de l'AEEC est plus large. À cet égard, soulignons que la part de contenu québécois dans les activités de l'AEEC est un sujet controversé qui a entraîné des frictions et des scissions parmi les membres de cette association (Mata 2001; Mata en entretien, 2006). Même si, en vertu du principe de la liberté universitaire, ces associations doivent être complètement indépendantes des mandats politiques de leurs institutions subventionnaires, dans la réalité, toutefois, les conflits d'intérêts sont loin d'être absents. 
Quant au développement des études canadiennes et québécoises en Espagne, selon Mata (2001 : 144), il résulte de l'équilibre «entre l'offre des différents organismes politiques ou associatifs et la demande des différents acteurs [espagnols] du monde de la formation et de la recherche». Ainsi, trois acteurs très différents, ayant des intérêts qui ne coïncident pas nécessairement, coopèrent pour l'accomplissement d'un projet commun dont ils anticipent des profits (symboliques ou économiques). Ces trois acteurs sont: les gouvernements fédéral et provincial, qui travaillent et investissent des fonds pour que le Canada (dont le Québec) soit (re)connu à l'étranger; l'AEEC et l'AIÉQ, financées par leurs gouvernements respectifs, qui veulent « recruter» des chercheurs d'autres pays pour qu'ils se joignent à leurs réseaux; enfin, une communauté universitaire espagnole, issue des études littéraires, qui manifeste un grand intérêt pour des littératures autres que la littérature française et qui perçoivent le Québec comme un foyer d'innovation, voire comme un «moteur de décolonisation» (Mata 2001: 149). À cela, il faut ajouter les généreux programmes de bourses de recherche et d'aide à la publication précédemment mentionnés, qui proviennent $\mathrm{du}$ gouvernement provincial, mais surtout du gouvernement fédéral (les bourses provenant du gouvernement fédéral dépassent largement en nombre et en sommes allouées celles du gouvernement du Québec) : étant donné que l'appui institutionnel à la recherche en Espagne est relativement limité, ces bourses suscitent un grand intérêt parmi les chercheurs espagnols.

En plus du dynamisme de ces réseaux associatifs, dynamisme qui a une incidence directe sur l'exportation de la littérature québécoise en Espagne, il convient de souligner l'importance du rôle individuel de certains de leurs membres (en grande partie des acteurs espagnols du monde de la recherche et de la formation), car ils participent directement à toutes les phases du transfert littéraire (sélection, production et réception) et sont présents dans la plupart des instances du champ littéraire proprement dit, soit (Boisclair 2004). Ainsi, on trouve parmi ces québécistes: (1) des traducteurs de la fiction québécoise (comme Lídia Anoll) et des éditeurs (comme Anik Lapointe); (2) des promoteurs et des organisateurs de tournées d'écrivains québécois (comme Carmen Mata); (3) des critiques universitaires (comme Carmen Mata, Áurea Fernández ${ }^{36}$ ); et (4) des professeurs de littérature québécoise (comme Carmen Fernández, Carmen Mata, Lídia Anoll, Áurea Fernández, etc.). Qui plus est, ces agents, normalement dotés d'un grand capital symbolique dans les champs culturels cibles, ont la capacité de mettre en rapport des réseaux de nature différente, par exemple des réseaux institutionnels et des réseaux éditoriaux, ou des réseaux éditoriaux et des réseaux médiatiques qui assurent la couverture de l'œuvre.

Faute d'espace, nous ne donnerons que deux exemples concrets. Lídia Anoll est une québéciste qui enseigne la littérature francophone (dont la littérature québécoise) à l'Université de Barcelone. Elle dirige des thèses portant sur la littérature québécoise et a publié un grand nombre d'articles dans ce domaine. C'est elle qui a traduit et présenté sur la scène catalane deux auteurs québécois, Anne Hébert et Jean-Paul Filion. Elle explique qu'après avoir travaillé de près avec l'auteur et avoir terminé la traduction catalane de Cap Tourmente (de Jean-Paul Filion), elle l'a proposée à la maison d'édition catalane Pagès, qui a accepté de la publier ${ }^{37}$. Quelques années plus tard, Àngels Santa, professeure à l'Université de Lleida et directrice de la collection «Lo marraco blau» chez Pagès, collection consacrée à la littérature (universelle) des femmes, lui a proposé de traduire un roman en français écrit par une femme. C'est 
Lídia Anoll qui a choisi Les chambres de bois (d'Anne Hébert), traduction catalane qu'elle a enrichie d'un "emballage» paratextuel très soigné qui présente l'auteure québécoise au public catalan. C'est donc l'intérêt d'une québéciste comme Lídia Anoll pour la littérature québécoise qui a motivé cette sélection d'œuvres à traduire. En même temps, cependant, le fait que Lídia Anoll détienne un certain capital symbolique (en raison de ses nombreuses publications sur les traductions d'auteurs français consacrés tels que Balzac), en plus de ses contacts chez Pagès (la plupart des collections de cette maison d'édition sont dirigées par des universitaires), a permis, par exemple, la présentation de ces œuvres dans le champ littéraire catalan.

Un autre exemple intéressant est celui d'Anik Lapointe, éditrice d'origine québécoise qui travaille pour la maison d'édition catalane La Magrana, maison qui a publié des œuvres d'auteurs québécois, comme Un dimanche à la piscine à Kigali (de Gil Courtemanche) et Le bruit des choses vivantes (d'Élise Turcotte). Connaissant aussi bien les champs littéraires catalan et espagnol que québécois, Anik Lapointe a elle-même proposé la traduction de ces œuvres (communication personnelle avec Anik Lapointe, le 11 décembre 2006). Si le choix de l'œuvre de Courtemanche n'a rien d'étonnant, car il s'agit d'un succès mondial en raison de sa thématique d'une brûlante actualité politique au moment de la publication, le choix du deuxième titre (qui n’a pas été traduit en espagnol, mais seulement en catalan) semble moins évident. $\mathrm{Au}$ critère de "valeur littéraire» de l'œuvre mis de l'avant par Anik Lapointe pour justifier ce choix, s'ajoute à n'en pas douter la subvention reçue du Conseil des Arts pour la traduire, ainsi que l'origine québécoise et la connaissance des affinités idéologiques entre les espaces sources et cibles de cette éditrice.

C'est donc bien la combinaison d'initiatives individuelles et institutionnelles qui est à la base de l'entreprise de la traduction de la fiction québécoise en Espagne et en Catalogne. L'existence d'un intermédiaire, ou de plusieurs, semble être ici une constante. L'autre constante, c'est que cet intermédiaire est connecté, d'une façon ou d'une autre, aux réseaux associatifs ou aux réseaux officiels présentés au fil de cet article.

\section{Vers une cartographie des réseaux d'échange littéraires Québec-Espagne-Catalogne}

Pour récapituler, deux grands types de réseaux sont à la base de la cartographie qui vient d'être tracée: d'une part, les réseaux institutionnels (bien structurés et hiérarchisés) et, d'autre part, les réseaux littéraires (flous et aux frontières poreuses). Dans le cas des réseaux institutionnels, deux types ont été présentés: les réseaux officiels (publics et qui relèvent de l'État) et les réseaux associatifs (semi-privés, c'est-à-dire, en partie financés par l'État). Dans le cas des premiers, deux grands réseaux opèrent parallèlement, mais en même temps de façon assez autonome, dans les espaces cibles: d'un côté, le réseau officiel fédéral et, de l'autre, le réseau officiel provincial. Pour ce qui est du réseau fédéral ressortent deux acteurs principaux: l'un représente le Canada en Espagne in situ, l'Ambassade du Canada en Espagne, et l'autre agit à partir des espaces sources, le Conseil des Arts. Pour le réseau provincial, le BQ Barcelone représente le Québec in situ (qui relève directement du ministère des Relations internationales, mais reçoit également des fonds d'autres ministères du gouvernement du Québec). Le BQ travaille également en coopération avec la SODEC et le CALC. 
Parmi les réseaux associatifs, ont été repérés des réseaux consacrés à l'édition (l'AELC, sur le plan fédéral, et l'ANEL, sur le plan provincial), ainsi que des réseaux universitaires (l'AEEC, sur le plan fédéral et l'AIÉQ, sur le plan provincial). En fait, on pourrait dire que les réseaux associatifs prolongent l'action des réseaux officiels dans des domaines spécifiques (le domaine de l'édition internationale pour les réseaux éditoriaux et le domaine de la recherche pour les réseaux universitaires).

Enfin, ont été mis au jour les réseaux littéraires, en principe indépendants des réseaux institutionnels et qui donnent lieu à des rapports peu structurés et imprévisibles entre les agents appartenant aux différents instances des champs littéraires sources et cibles. Il s'agit de rapports basés sur des affinités littéraires, idéologiques ou autres: par exemple, entre les éditeurs québécois et les éditeurs catalans ou espagnols, entre les écrivains des aires sources et cibles, entre les éditeurs des aires cibles et les écrivains des aires sources, etc. Une classification plus précise et découpée reste cependant à faire.

\section{En guise de conclusion}

Nous avons tenté de répondre à la question de savoir par quels moyens le Québec peut manifester son existence en Espagne et en Catalogne. Pour ce faire, nous avons présenté une cartographie composée de divers réseaux, des plus institutionnalisés aux plus informels, mais nous avons également souligné la part des initiatives individuelles, c'est-à-dire des agents-clés, des nœuds, qui assurent la connexion entre ces réseaux de nature diverse. Nous avons voulu montrer que très souvent l'action de ces réseaux permet à la fiction québécoise de franchir les frontières linguistiques et de surmonter les obstacles à la diffusion d'une littérature minoritaire. Cependant, nous avons également mentionné que l'action de ces réseaux ne suffit pas pour répondre à la question posée dans cet article. Il faut en effet que la ou les communautés cibles portent un intérêt quelconque à la culture source et ses productions culturelles. Cet article montre donc qu'à l'heure d'aborder les échanges littéraires, on a tout intérêt à porter le regard non seulement vers la culture cible, mais aussi vers la culture source, ce qui revient à inverser la perspective traditionnelle. L'étude des stratégies d'exportation et de promotion de la littérature québécoise en Espagne axée sur les multiples agents et acteurs, les processus complexes de coopération, leurs intermédiaires, etc., invite à relativiser les hypothèses fonctionnalistes. Elle permet de mettre en relief la nature collective et coopérative de l'entreprise de traduction, non seulement du processus traductif en tant que tel, mais aussi de son avant et de son après. À ce titre, une cartographie des réseaux offre l'avantage de bien représenter la complexité des échanges littéraires, comme d'autres traductologues l'ont déjà relevé (Pym 1998). La catégorisation des réseaux proposée ici pourrait d'ailleurs être transposée à d'autres contextes.

\section{NOTES}

1. Pour expliquer le concept de «transfert culturel», nous empruntons la définition opératoire adoptée par la Chaire de recherche du Canada en transferts littéraires et culturels sous la direction de Walter Moser. On le définit comme suit: «[L]e transport de matériaux culturels d'une culture à une autre. Il y a donc déplacement et transport, franchissement d'une distance, passage d'un seuil. Au point de départ, il y a sélection de ce qui sera transporté, prélèvement, décontextualisation; au point d'arrivée, réinsertion, recontextualisation. Le matériau transféré se transforme dans le 
processus, puisque son identité et son sens sont fonction d'une relation à un contexte» (<http:// www.socialsciences.uottawa.ca/transferts/fra/intro.asp $>$, dernières modifications: 2007/02/14).

2. Quand nous employons le terme «agents» dans le cadre de cet article, nous faisons référence à des personnes. Nous emploierons le terme «acteurs » pour nous référer à des organismes officiels ou à des associations privées ou semi-privées composées de plus d'un individu.

3. C'est-à-dire dans la manière dont ils étiquettent et introduisent ces traductions dans les espaces cibles.

4. Les institutions supra-littéraires désignent les divers «agents actifs dans les pratiques littéraires sans que pour autant celles-ci constituent leur objet principal [...] Telles sont l’École, l'Église [...], tout l'appareil des médias, les festivals, les ministères, et maisons de la culture...» (Viala, 1990: 122).

5. Le concept d'«institutions» est employé dans cet article en tant que structures sociales organisationnelles, en tant qu'instances qui «élèvent des pratiques du rang d'usages à celui de valeurs par un effet de pérennisation (et qui, ce faisant, s'érigent elles-mêmes en autorités), et les valeurs ainsi établies» (Viala, 1990: 120). Sauf indication contraire, lorsque nous parlons d'«institutions », nous nous référons à des institutions supra-littéraires et rarement à des institutions littéraires en tant que telles. De même, quand nous faisons allusion aux «réseaux institutionnels», nous faisons référence à des réseaux institutionnels supra-littéraires. Nous employons le mot institutionnel pour souligner la nature structurée et formelle de ces réseaux, tandis que par la formule «réseaux littéraires», nous mettons en relief le caractère flou, vague et imprévisible de ces derniers.

6. Par «Espagne» ou «champ culturel espagnol», nous entendons l'espace où circulent et sont «consommées» les productions culturelles en espagnol (originales et traduites) - sans oublier qu'il ne s'agit pas d'un espace homogène, mais d'un espace au sein duquel cohabitent différents noyaux identitaires régionaux plus ou moins marqués. Cet espace inclut aussi la Catalogne, où les productions culturelles mentionnées circulent et sont également consommées, étant donné que l'espagnol (ou castillan) y est, à côté du catalan, une langue officielle. Or, il existe en Catalogne une histoire, une langue et une production littéraire catalophone. Par conséquent, quand nous parlons de «la Catalogne», ou du «champ culturel catalan», nous faisons référence à cet espace de circulation et de consommation de productions culturelles en catalan (originales et traduites), qui se veut différent de l'espace culturel espagnol.

7. Citons à titre d'exemple le numéro de la revue The Translator paru en 2005 et dirigé par Moira Inghilleri intitulé Bourdieu and the Sociology of Translation and Interpreting, ou, antérieurement, le numéro spécial des Actes de la recherche en sciences sociales (2002), sous la direction de Johan Heilbron et Gisèle Sapiro, intitulé Traduction: les échanges littéraires internationaux. À cela il faut ajouter les travaux de Gouanvic (1999), Simeoni (1998) et Wolf (2002), pour ne citer que les plus connus.

8. Cette insistance de Bourdieu sur le concept de lutte pourrait s'expliquer à la lumière du contexte français du sociologue. Selon Exler (1999: 2), le système français, «qui valorise uniquement les individus et leurs talents et tente de détecter les meilleurs, engendre une forme particulière d'organisation sociale. L'individu ne peut y jouer que des jeux à somme nulle: pour les gagnants, le gain sera maximal et se mesure par la perte, elle aussi totale, des perdants». Selon Exler (1999: 2): «La disparité radicale entre les gagnants et les perdants alimente le caractère non coopératif de l'organisation sociale française», ce qui incite au combat solitaire entre les agents, d'où peut-être la vision agonistique du champ de Bourdieu.

9. Latour est lui-même conscient de l'imprécision du concept de « réseau »: «The word network is so ambiguous that we should have abandoned it long ago » (2005: 129). Toutefois, il affirme ironiquement: «I find it best to use the most general, the most banal, even the most vulgar repertoire so that they will be no risk of confusing the actors' own prolific idioms. Sociologists of the social, as a rule, do just the opposite. They are keen to produce precise, well chosen, sophisticated terms for what they say the actors say» (Latour, 2005: 29).

10. Soit un instrument de description et un objet à décrire (Lacroix, 2006).

11. Sur ce point, notre emploi du concept de «réseau» est plus proche de celui de Bourdieu, qui voit ce concept en termes de «capital social» et définit ce dernier comme «l'ensemble des ressources actuelles ou potentielles qui sont liées à la possession d'un réseau de relations durables plus ou moins institutionnalisées d'interconnaissance et d'inter-reconnaissance» (Bourdieu, 1980: 2). Il ne faut pas oublier, cependant, que pour Bourdieu le capital social n'est que secondaire par rapport à d'autres espèces de capital (notamment le capital culturel et économique) dont la distribution déterminera la position d'un agent dans le champ. Le capital social ne peut pas être dissocié des 
autres types de capital et l'étude de ces «réseaux de relations effectives» liés à l'accumulation de capital social occupe une place très secondaire dans la théorie des champs (voir note 16). Il convient également de souligner que pour nous, le réseau n'est pas seulement un objet à décrire, mais aussi un instrument de description.

12. Pour une bonne illustration de l'emploi métaphorique du concept de réseau chez Callon et Latour, voir le cas des coquilles Saint-Jacques (Callon, 1986).

13. L'approche relationnelle qu'implique la théorie des champs postule «que les individus se définissent les uns par rapport aux autres indépendamment des relations effectives entre eux (qui peuvent bien sûr exister ou se concrétiser à un moment donné, mais qui peuvent aussi masquer les relations objectives: ainsi une relation de forte concurrence entre deux individus est souvent l'expression de la proximité des positions qu'ils occupent)» (Sapiro, 2006: 47).

14. Les questionnaires créés pour ces entretiens contenaient des questions visant à obtenir des renseignements factuels (par exemple, quel est le budget annuel consacré au programme de subventions à la traduction internationale du Conseil des Arts du Canada?) et d'autres visant à prendre connaissance de l'avis de l'agent en tant que représentant d'un organisme sur un aspect particulier (par exemple, comment concilier l'idée d'appuyer financièrement une œuvre qui exhibe une spécificité culturelle particulière - en l'occurrence la spécificité québécoise - et celle de transmettre une représentation «unifiée» du Canada?). Bien entendu, les questionnaires varient d'un entretien à l'autre, car les agents interviewés représentent des organismes différents. Nous avons interviewé jusqu'à ce jour: Laura Ballesteros, attachée culturelle de l'Ambassade du Canada en Espagne (le 16 juin 2006); María Jesús Bronchal, attachée aux affaires publiques et culturelles du BQ Barcelone (le 21 juin 2006); Catherine Montgomery, agente du Service des lettres et de l'édition du Conseil des Arts du Canada (le 18 octobre 2006); et Carmen Mata, professeure à l'Universidad Autónoma de Madrid et représentante de l'AIÉQ en Espagne (15 juin 2006). Nous avons donc rencontré les agents-clés appartenant aux instances supra-littéraires contribuant à l'entreprise de la traduction de la fiction québécoise en Espagne. Nous avons aussi échangé une correspondance avec Beatriu Cajal, agente du Service des lettres et de l'édition de l'Insitució de les Lletres Catalanes, et Marisa Calés, secrétaire-trésorière de l'Asociación Española de Estudios Canadienses. Seront ultérieurement interviewés les éditeurs espagnols et catalans qui ont décidé de publier ces traductions.

15. C’est-à-dire sa capacité à servir d'intermédiaire entre les autres acteurs du réseau.

16. À cet égard, notons que l'Espagne et la Catalogne figurent dans le cadre de la nouvelle politique internationale du Québec, rendue publique le 24 mai 2006, comme partenaires prioritaires. De même, dans la section consacrée à la promotion de l'identité et de la culture du Québec, le soutien à la traduction en langue espagnole représente également une action importante à poursuivre $<$ http://www.mri.gouv.qc.ca/fr/pdf/Politique.pdf $>$.

17. Par exemple, dans la politique étrangère canadienne de 1970, Politique étrangère au service des Canadiens, qui a été en vigueur jusqu'en 1995, on parle du «Renouveau du Canada français» immédiatement après une section intitulée «L'influence américaine, un sujet de préoccupation grandissante». De même, le rapport Tremblay de 1956 avait déjà insisté sur l'importance du fait francophone en tant qu'élément qui distingue le Canada des États-Unis. En réalité, ces exemples ne constituent pas des exceptions: on peut observer ce discours sur l'emploi du fait francophone non seulement dans des documents du passé récents, mais dans des textes actuels (officiels ou non).

18. Comme avec Francisco Torres Montreal, intéressé par la littérature québécoise et traducteur d'Hélène Dorion et de Louise Dupré, et bénéficiaire d'une bourse de recherche du MAÉCI en 2006.

19. À la suite d'une rencontre organisée par le personnel du consulat du Canada à Barcelone (et en coopération avec l'Ambassade du Canada à Madrid) entre les éditeurs catalans et la directrice générale de l'AELC, l'Association des éditeurs de Catalogne a invité l'AELC à envoyer une délégation d'éditeurs canadiens à Liber 2004. C'est précisément une subvention du Generalitat de Catalogne qui a aidé à payer les coûts de cette participation. En tout, 11 éditeurs ont participé, exposant aux stands collectifs organisés par l'AELC et Québec Édition. Le consul canadien, Khawar Nasim, et la directrice du Bureau du Québec à Barcelone, Michelle Bussières, ont offert conjointement une réception à l'intention des éditeurs canadiens, québécois, catalans et espagnols (AELC, 2005): <http://www.aecb.org/fra/about-us.asp?id=4>.

20. L’AELC représente aussi l'industrie éditoriale québécoise, mais celle-ci est plus directement représentée par l'ANEL (Association nationale des éditeurs de livres). L'Ambassade (Ballesteros, 2006) encourage aussi le rapprochement entre les maisons d'édition espagnoles et québécoises, même s'il est évident qu'elle ne le fait pas de manière aussi intensive que le BQ Barcelone. 
21. On peut jeter un coup d'œil à la liste de traductions fournie par l'Ambassade du Canada en Espagne sur son site <http://www.international.gc.ca/canada-europa/spain/libros-es.asp >. Cette dernière liste n'étant pas exhaustive, nous ne nous attarderons pas à fournir des données chiffrées précises comme dans le cas des livres québécois.

22. <http://www.conseildesarts.ca/subventions/politiques_subventions/gq127234205403281250.htm>.

23. Il s'agit de La verdadera historia del perro de Clara Vic et Víctor (C. Duchesne), de Casiopea o el verano polaco/Cassiopea o l'estiu polonès. (M. Marineau); et de Raymond Plante, La màquina de la bellesa/La máquina de la belleza; El récord de Philibert Dupont/El rècord d'en Philibert Dupont; El último pasmarote/L'ultim estaquirot; avec M. Melançon El gos salsitxa i els lladres de diamants; enfin, Millones por una canción/ Milions per una cançó (A. Vanasse).

24. Il s'agit de El libro de Emma (M.-C. Agnant); Gatuperios (Yves Beauchemin); La ingratitud (Y. Chen); Un diumenge a la piscina a Kigali (Gil Courtemanche); Cómo hacer el amor con un negro sin cansarse (Dany Laferrière); La nena que li agradaven massa els llumins (G. Soucy); et El soroll de les coses vives (E. Turcotte).

25. María Jesus Bronchal (2006) insiste sur le fait que le mandat initial du BQ Barcelone n'était pas politique: «On n'est pas venus ici pour faire de la politique ou pour vendre du souverainisme. Ce que les gens pensent, c'est bien différent...» Cependant, le fait que le BQ se soit installé à Barcelone (plutôt qu'à Madrid) et qu'il ait été créé sur la proposition de Jordi Pujol au moment où le Parti québécois était au pouvoir pourrait indiquer que l'aspect politique n'était pas tout à fait absent de cette initiative, même s'il est également vrai que le BQ a maintenu le même mandat (commercial) plus tard avec un autre gouvernement, en l'occurrence le gouvernement libéral.

26. Québécoises tout simplement parce qu’elles ont été écrites par des auteurs québécois. On pourrait alléguer que la dimension «québécoise» de ces œuvres est très secondaire et qu'elle ne constitue donc pas un facteur important dans leur exportation ou leur réception critique. Cela est vrai si l'on donne à "québécois» un sens restreint et plutôt conventionnel. D’un autre côté, on pourrait dire à la limite qu'elles présentent une dimension «québécoise» dans le sens que, aussi bien l'internationalisme de l'œuvre de Courtemanche que la voix revendicative des femmes présente dans Putain, voix associée à l'écriture au féminin, d'ailleurs perçue comme une écriture spécifique au contexte littéraire québécois, font partie de l'«image de marque» du Québec que le gouvernement de cette province veut projeter. Les entrevues et la correspondance avec les éditeurs permettront de savoir à quel point les éditeurs catalans qui ont pris l'initiative de faire traduire ces œuvres ont été influencés par l'image que le Canada ou le Québec souhaitent projeter d'eux-mêmes à l'étranger.

27. Tandis que les traductions catalanes de notre corpus tendent à mettre en relief la spécificité québécoise (Córdoba Serrano, à paraître.), dans le cadre des traductions espagnoles, la spécificité québécoise n'est pas nécessairement au premier plan, comme c'est généralement le cas de l'élément canadien-français.

28. Il s'agit plus précisément de El libro de Emma de M.-C. Agnant et de La ingratitud de Y. Chen, mais aussi de Dany Laferrière dans Cómo hacer el amor con un negro sin cansarse et de ¿Esa granada en la mano del joven negro es un arma o una fruta?, d'Émile Ollivier dans Pasos, et, enfin de Fuera Perros et Una aurora boreal de Folch-Ribas. Folch-Ribas, un Catalan exilé en France puis au Québec, constitue un cas à part, car sa traduction ne sera pas considérée en Espagne comme une "voix venue d'ailleurs», mais comme une manifestation de la littérature de l'exil espagnol.

29. «Dany Laferrière enseña cómo hacer el amor con un negro sin cansarse», La Vanguardia, le 19 novembre 1997.

30. «Escritoras de culturas remotas conquistan el mercado español: africanas, indias, caribeñas y chinas se convierten en el filón de varias editoriales », El País, le 26 décembre 1999.

31. Trois des sept œuvres de l'écriture migrante québécoise traduites en Espagne ont été publiées dans des maisons d'édition, telles que Txalaparta et Ediciones El Cobre, dont la mission principale est de promouvoir la diversité et les voix venues d'ailleurs. Le reste des maisons d'édition font preuve dans leur catalogue d'une certaine ouverture à la diversité culturelle, sans que cette littérature n'y représente pour autant la partie centrale. Parfois, comme dans le cas de La ingratitud de Y. Chen, le choix est tout simplement arbitraire: Lémeac, l'éditeur de l'original avait envoyé un catalogue à la maison d'édition, et cette œuvre a attiré l'attention de la directrice éditoriale, Sigrid Kraus (communication personnelle avec Alix Laveugle, maison d'édition Salamandra, le 22 février 2007). Il est possible, cependant, que l'arrivée en grand nombre d'immigrants chinois en Espagne et l'importance qu'acquiert la Chine dans l'imaginaire des Espagnols aient exercé une certaine influence sur la sélection de cette œuvre. 
32. Marta Tey (Ediciones el Cobre) a répondu (communication personnelle, le 26 janvier 2007) que 2500 exemplaires seulement de ¿Esa granada en la mano del joven negro es un arma o una fruta? de Dany Laferrière avaient été vendus, mais que Laferrière est un des auteurs préférés de cette maison d'édition, qui compte bien continuer à le publier. La maison d'édition Salamadra nous a indiqué un chiffre de vente très comparable pour La ingratitud de Y. Chen. Alix Laveugle, de cette même maison d'édition, juge ce volume de ventes très limité. Il faut dire que c'est cette dernière qui publie la traduction espagnole de Harry Potter...

33. Bien entendu, non seulement les traductions de l'écriture migrante québécoise, mais aussi celles provenant d'autres champs littéraires. Sur ce point, voir Mata (2004).

34. Plus précisément, les ministères (1) des Relations internationales et (2) de la Culture et des Communications du gouvernement du Québec.

35. Particulièrement le MAÉCI.

36. Cette forme de critique - en contraste avec la critique journalistique - est de grande importance pour la réception de la littérature québécoise, car comme l'indique Fernández (2004: 57): «Les lecteurs espagnols qui connaissent le mieux la production canadienne appartiennent au domaine universitaire et présentent, par conséquent, des traits propres et des expectatives particulières».

37. Communication personnelle avec Lídia Anoll, le 30 septembre 2006.

\section{RÉFÉRENCES}

ANDRÉS-SuÁREZ, I. (2004) : «Inmigración y literatura española actual: las voces del Estrecho », España contemporánea: Revista de literatura y cultura, 17-1, pp. 7-24.

Boisclair, I. (2004): Ouvrir la voie/x. Le processus constitutif d'un sous-champ littéraire féministe au Québec (1960-1990), Québec, Nota bene.

Bourdieu, P. (1980): "Le capital social: notes provisoires», Actes de la recherche en sciences sociales, 31, pp. 2-3.

Bourdieu, P. (1994): Raisons pratiques: sur la théorie de l'action, Paris, Seuil.

Bourdieu, P. (1998): Les règles de l'art. Genèse et structure du champ littéraire, Paris, Seuil.

Bourdieu, P. (2002): «Les conditions sociales de la circulation internationale des idées», Actes de la recherche en sciences sociales, 145, pp. 3-8.

Brisset, A. (1990): Sociocritique de la traduction. Théâtre et altérité au Québec (1968-1988), Longueuil, Le Préambule.

Buzelin, H. (2005): «Unexpected Allies: How Latour's Network Theory Could Complement Bourdieusian Analyses in Translation Studies», Inghilleri, M. (ed.), Bourdieu and the Sociology of Translation and Interpreting, numéro spécial, The Translator, 11-2, pp. 193218.

Callon, M. (1986): «Éléments pour une sociologie de la traduction, la domestication des coquilles Saint-Jacques et des marins-pêcheurs dans la baie de Saint-Brieuc», L'année sociologique, 36, pp. 169-208.

Callon, M. et B. Latour (dir.) (1991): La Science telle qu'elle se fait: anthologie de la sociologie des sciences de langue anglaise, Paris, Éditions La Découverte.

Casanova, P. (1999): La République mondiale des lettres, Paris, Seuil.

Casanova, P. (2002): «Consécration et accumulation de capital littéraire: la traduction comme échange inégal», Heilbron, J. et G. Sapiro (dir.), Traductions: les échanges littéraires internationaux, numéro spécial, Actes de la recherche en sciences sociales, 144, pp. 7-20.

Claisse, F. (2006) : «De quelques avatars de la notion de réseau en sociologie», in DE MARneffe, D. et B. Denis (dir.), Les Réseaux littéraires, Bruxelles, le Cri, pp. 21-43.

Córdoba Serrano, M. S. (à paraître): «Adieu coureurs des bois et filles du roi: Québecs imaginaires en Espagne», dans Espagnes imaginaires (sous la direction de C. MAтA), Montréal, Hurtubise HMH.

DuвоIs, J. (1978): L'institution de la littérature: introduction à une sociologie, Bruxelles, Editions Labor.

Even-Zohar, I. (1990): «Laws of Literary Interference», Polysystem Studies [=Poetics Today $11: 1]$, pp. 53-72. 
ExLER, A. (1999) : «La majorité désarmée. Coopération et concurrence dans la société française», Le Banquet, 14, pp. 1-8.

Fernández Rodríguez, A. (2004): «La création littéraire canadienne d'expression française traduite en Espagne. Une culture peu connue», dans Laliberté, R. et D. Monière (dir.), Actes du Colloque européen Le Québec au miroir de l'Europe, Chapitre I - Langues, cultu-

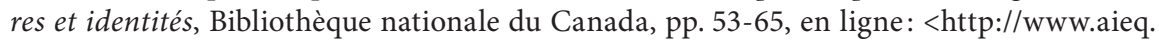
qc.ca/nouveautes/actes_aieq.doc $>$.

Gellner, E. (1989): Nations et nationalisme (traduit de l'anglais par B. Pineau), Paris, Payot.

GouAnvic, J.-M. (1999): Sociologie de la traduction: la science-fiction américaine dans l'espace culturel français des années 1950, Arras, Artois Presses Université.

Gutiérrez-Haces, M. T. (2003): «Identity and Otherness in Canadian Foreign Policy», dans The Canadian Distinctiveness into the $X X^{s t}$ century/La disctinction canadienne au tournant $d u X X^{e}$ siècle, (ed. Gaffield, C. and K. L. Gould), Ottawa, les Presses de l'Université d'Ottawa, pp. 231-250.

Hermans, T. (1999): Translation in Systems: Descriptive and Systemic Approaches Explained, Manchester, St Jerome.

Inghilleri, M. (2005): "The Sociology of Bourdieu and the Construction of the 'Object' in Translation and Interpreting Studies», INGHILleri, M. (ed.), «Bourdieu and the Sociology of Translation and Interpreting», numéro spécial, The Translator, 11-2, pp. 125-145.

LAcroix, M. (2003) : «Littérature, analyse de réseaux et centralité: Esquisse d'une théorisation du lien social concret en littérature», Recherches sociographiques, 44-3, pp. 474-497.

LAcroix, M. (2006): «Points, triades, trous ou comment décrire les réseaux littéraires. Le cas des relations entre Léo-Paul Desrosiers et les éditions Gallimard», Les Réseaux littéraires (ed. De Marneffe, D. et B. Denis), Bruxelles, le Cri, pp. 201-224.

LAtour, B. (2005): Reassembling the Social: an Introduction to Actor-Network Theory, Oxford, Clarendon.

Mata Barreiro, C. (2001): «Les études québécoises en Espagne: des indices d'une maturité», Globe. Revue internationale d'études québécoises, 4-2, pp. 143-159.

Mata Barreiro, C. (2004): «La réception de la littérature migrante au Québec, dans l'Europe francophone et en Espagne», dans Laliberté, R. et D. Monière (dir.), Actes du colloque européen Le Québec au miroir de l'Europe Chapitre I - Langues, cultures et identités, pp. 8998, en ligne: <http://www.aieq.qc.ca/nouveautes/actes_aieq.doc>.

Oeding, B et L. von Flotow (2005): «Soft Diplomacy, Nation Branding, and Translation: Telling Canada's 'Story' Globally", Translation. Reflections, Refractions, Transformations, St-Pierre, P. and P. C. Kar (eds.), New Delhi, Pencroft International, pp. 173-193.

Potter, E. (1996/1997): «Niche Diplomacy as Canadian Foreign Policy», International Journal, 52-1, p. 25.

Potter, E. (2002): «Canada and the New Public Diplomacy», Discussion Papers in Diplomacy 81, pp. 1-19, en ligne: <www.clingendael.nl/publications/2002/20020700_cli_paper_dip_ issue81.pdf>.

Potter, E. (2004): «Branding Canada: the Renaissance of Canada’s Commercial Diplomacy», International Studies Perspectives, 5, pp. 55-60.

Рyм, A. (1998): Method in Translation History, Manchester, St. Jerome Publishing.

Sapiro, G. (2006): «Réseaux, institution(s) et champ», in De Marneffe, D. et B. Denis (dir.), Les Réseaux littéraires, Bruxelles, le Cri, pp. 44-59.

SAUL, J. R. (1994) : «Culture and Foreign Policy», le 30 août 1994, en ligne: <http://www.mediaawareness.ca/english/resources/articles/sovereignty_identity/culture_policy.cfm $>$.

Simeoni, D. (1998): «The Pivotal Status of the Translator's Habitus», Target, 10-1, pp. 1-39.

Toury, G. (1980): In search of a Translation Theory, The Porter Institute for Poetics and Semiotics, Tel Aviv University.

Toury, G. (1995): Descriptive translation studies and beyond, Amsterdam/Philadelphia, John Benjamins Publishing Company. 
Viala, A. (1990): «L'histoire des institutions littéraires», in BéHAR, H. et R. Fayolle (dir.), L'histoire littéraire aujourd'hui, Paris, Armand Colin, pp. 118-128.

Wolf, M. (2002): «Translation Activity between Culture, Society and the Individual: Towards a Sociology of Translation", in Harvey, K. (ed.), CTIS Occasional Papers, volume 2, Manchester, UMIST, pp. 33-43.

\section{ENTREVUES}

Entrevue avec Laura Ballesteros, attachée culturelle de l'Ambassade du Canada en Espagne, 16 juin 2006.

Entrevue avec María Jesús Bronchal, attachée aux affaires publiques et culturelles du Bureau du Québec à Barcelone, 21 juin 2006.

Entrevue avec Carmen Mata, professeure de l'Universidad Autónoma de Madrid en Espagne, 15 juin 2006.

Entrevue avec Catherine Montgomery, agente du Service des lettres et de l'édition du Conseil des Arts du Canada, 18 octobre 2006.

\section{ANNEXE}

\section{Liste de traductions espagnoles et catalanes}

Remarque: l'astérisque indique les traductions catalanes

Agnant, Marie-Célie, El libro de Emma, Tafalla, Txalaparta, 2003 (Traduction en espagnol de José Antonio Jimeno. Original français: Le livre d'Emma, Montréal, les éditions du RemueMénage, 2001).

ArCAN, Nelly, Puta, Barcelona, Seix-Barral, 2002. Rééditée chez Planeta en 2005 (Traduction en espagnol de Guadalupe Ramírez. Original français: Putain, Paris, Seuil, 2001).

${ }^{\star}$ ArCAn, Nelly, Puta, Barcelona, Columna, 2002 (Traduction en catalan de Pau Joan Hernàndez. Original français: Putain, Paris, Seuil, 2001).

BeAUChemin, Yves, Gatuperios, Madrid, Alianza, 1989, (Traduction en espagnol de María Teresa Gallego Urrutia et de María Isabel Reverte Cejudo. Original français: Le Matou, Montréal, Éditions Québec/Amérique, 1981).

BRIÈRE, Paule, Leoncito tiene dos casas, Madrid, Edelvives, 2003, (Traduction en espagnol de P. Rozarena. Original français: P'tit lion a deux maisons, Paris, Père Castor Flammarion, 2000).

BRossard, Nicole, Barroco al alba, Barcelona, Seix Barral, 1998. (Traduction en espagnol de Pilar Giralt Gorina. Original français: Baroque d'aube, Montréal, L’Hexagone 1995.).

BRÛLÉ, Michel. El niño que quería dormir, Barcelona, Obelisco, 2004 (Traduction en espagnol de Javier Aguirre. Original Français, L'enfant qui voulait dormir, Paris, Grasset-Jeunesse, 2005).

CHeN, Ying, La ingratitud, Barcelona, Emecé, 1998. (Traduction en espagnol de María Luz García de la Hoz. Original français: L’ingratitude, Montréal, Leméac, 1995).

Côté, Denis, El parque de los sortilegios, Zaragoza, Edelvives, 2000. (Traduction en espagnol de Susana Vázquez Jiménez. Original français: Le parc aux sortilèges, Montréal, La courte échelle, 1994).

Côté, Denis, Rehenes del terror, Zaragoza, Edelvives, 2001. (Traduction en espagnol de Susana Vázquez Jiménez. Original français: Les otages de la terreur, Montréal, La Courte échelle, 1998).

Courtemanche, Gil, Un domingo en la piscina en Kigali, Barcelona, Emecé, 2003. (Traduction en espagnol de María José Furió. Original français: Un dimanche à la piscine à Kigali, Montréal, Boréal, 2000).

${ }^{\star}$ Courtemanche, Gil, Un diumenge a la piscina a Kigali, Barcelona, La Magrana, 2003. (Traduction en catalan de Anna Casassas. Original français: Un dimanche à la piscine à Kigali, Montréal, Boréal, 2000). 
Davidts, Jean-Pierre, Reencuentro con «El Principito», Barcelona, Ediciones B, 1999. (Traduction en espagnol de Teresa Clavel. Original français: Le petit prince retrouvé, Montréal, Les Intouchables, 1993).

Demers, Dominique, El viejo Tomás y la pequeña hada, Barcelona, Juventud, 2003. (Traduction en espagnol d'Élodie Bourgeois. Original français: Vieux Thomas et la petite fée, Saint-Lambert, Dominique et cie, 2000).

${ }^{\star}$ Demers, Dominique, El vell Tomàs i la petita fada, Barcelona, Juventud, 2003. (Traduction en espagnol de Teresa Farrán. Original français: Vieux Thomas et la petite fée, Saint-Lambert, Dominique et cie, 2000).

DuCheSne, Christiane, La verdadera historia del perro de Clara Vic, Barcelona, Edebé, 1992. (Traduction en espagnol d'Amada Perelló. Original français : La vraie histoire du chien de Clara Vic, Montréal, Éditions Québec/Amérique, 1990).

Duchesne, Christiane, Víctor, Barcelona, Edebé, 1993. (Traduction en espagnol de Dominique Delandre. Original français: Victor, Éditions Québec/Amérique, 1992).

*Filion, Jean-Paul, Del cap tempesta al cap de Joia, Lleida, El timó. Pagès Editors, 1993. (Traduction en catalan de Lídia Anoll Vendrell. Original français: Cap Tourmente, Montréal, Leméac, 1980).

FISHER, Mark, El regalo del millonario: un cuento sobre el trabajo y el amor, Barcelona, Gedisa, 2002. (Traduction en espagnol de Rosa Solà Maset. Original français: Le Cadeau du Millionnaire: un conte sur le travail et l'amour, Montréal, Éditions Québec/Amérique, 1998).

Fisher, Mark, El testamento del millonario: un relato sobre el arte de triunfar y ser feliz, Barcelona, Gedisa, 2003. (Traduction en espagnol de Jose Luis Sánchez. Original français: Le testament du Millionnaire: sur l'art de réussir et d'être heureux, Saint-Hubert, Éditions Un Monde différent, 2002).

FolCh-Ribas, Jacques, Una aurora boreal, Madrid, Selections de Reader's Digest. Biblioteca de Selecciones, 1978. (Traduction en espagnol de Miguel Mur, et al. Original français: Une aurore boréale, Paris, Laffont, 1974).

Folch-RibAs, Jacques, Fuera perros, Barcelona, Laia. Collect. Los Extraordinarios, 1987. Réédité dans Laia. Collect. Alfa 7, 1988. (Traduction en espagnol de Jordi Marfà. Original français: Dehors les chiens Acropole, Paris, Acropole, 1986).

GAgnon, Cécile, Un barril en el mar, Madrid, SM\&B, 1991. Réédité à Barcelona: Bayard, 2001. (Traduction en espagnol de Luis Prensa et illustrations de Catherine Munière. Original français: Une lettre dans la tempête, Paris, Centurion, 1989).

GAgnon, Cécile, El arco iris, Barcelona, Milán, 1991. (Traduction en espagnol: L’arc-en-ciel Toulouse, Milan, 1991).

GAgnon, Cécile, El disfraz, Barcelona, Milán, 1991. (Traduction en espagnol. Original français: Le déguisement, Toulouse, Milan, 1991).

Gagnon, Cécile, La pelea, Barcelona, Milán, 1991. (Traduction en espagnol. Original français: La dispute, Toulouse, Milan, 1991).

Gagnon, Cécile, El para-viento, Barcelona, Milan, 1991. (Traduction en espagnol. Original français: Le paravent, Toulouse, Milan, 1991).

Gagnon, Cécile, Demasiado ruido, Barcelona, Milan, 1991. (Traduction en espagnol. Original français: Trop de bruit, Toulouse, Milan, 1991).

Gagnon, Cécile, La pista de patinaje, Barcelona, Milan, 1991. (Traduction en espagnol. Original français: La patinoire, Toulouse, Milan, 1991).

${ }^{\star}$ Gagnon, Cécile, L’Arc de Sant Martí, Barcelona, Milan, 1991. (Traduction en catalan: L'arc-enciel, Toulouse, Milan, 1991).

${ }^{\star}$ Gagnon, Cécile, El Paravent, Barcelona, Milan, 1991. (Traduction en catalan. Original français: Le paravent, Toulouse, Milan, 1991).

*Gagnon, Cécile, La Disfressa, Barcelona, Milan, 1991. (Traduction en catalan. Original français: Le déguisement, Toulouse, Milan, 1991). 
${ }^{*}$ Gagnon, Cécile, La Baralla, Barcelona, Milan, 1991. (Traduction en catalan. Original français: La dispute Toulouse, Milan, 1991).

${ }^{\star}$ GAGNon, Cécile, Massa soroll, Barcelona, Milan, 1991. (Traduction en catalan. Original français: Trop de bruit, Toulouse, Milan, 1991).

${ }^{*}$ GAGNon, Cécile, La Pista de patinatge, Barcelona, Milan, 1991. (Traduction en catalan. Original français: La patinoire, Toulouse, Milan, 1991).

Gagnon, Madeleine, Las mujeres dan la vida, los hombres la quitan, Barcelona, Crítica, 2001. (Traduction en espagnol de Silvia Furió. Original français: Les femmes et la guerre, Montréal, VLB, 2000).

GAUTHIER, Gilles, El gran problema del pequeño Marcos, Zaragoza, Edelvives, 2002. (Traduction en espagnol de P. Rozarena et illustrations de Pierre-André Derome. Original français: Le gros problème du petit Marcus, Montréal, La courte échelle, 1992).

HaCikyan, Agop J. et Soucy, Jean-Yves, Un verano sin alba. Barcelona, Emecé, 1997. (Traduction en espagnol de Amanda Forns. Original français: Un été sans aube Montréal, Libre Expression, 1991).

HARveY, André. El último perdón, Barcelona, Luciérnaga, 2001 (Traduction espagnole de Blanca Ávalos. Original français: L’ultime pardon, Boucherville, Éditions de Mortagne, 1993).

HeberT, Anne, Kamouraska, Barcelona, Edición de Plaza \& Janés, 1972. (Traduction en espagnol de J. María Martinez Monasterio Original français: Kamouraska). Réédité en 1977 chez Plaza \& Janés et chez Esplugas de Llobregat, G.P., [1 ${ }^{\text {ere }}$ ed.], 1977. Original français: Kamouraska, Paris, Édition du Seuil, 1970).

`HÉBERT, Anne, Les cambres de fusta, Lleida, Pagès editors, 2001, col.1 «Lo Marroco blau» (Traduction en catalan de Lídia Anoll. Original français: Les chambres de bois Paris, Édition du Seuil, 1958).

HéBerT, Bruno, ¡Yo no fui, lo juro!, Barcelona, Andrés Bello, 2000 (Traduction en espagnol de Pierre Jacomet. Original français: C'est pas moi, je le jure, Montréal, Boréal, 1998).

Hémon, Louis, María Chapdelaine, Madrid: Rivadeneyra, cop. 1923, Réédité Rivadeneyra en 1929, chez Nausica en 1942 et en 1945, chez José Janés Editor en 1950; Ariel, 1952; chez Plaza \& Janés en 1975. (Traduction espagnole de A. Hernández Catá. Original français: Maria Chapdelaine, Paris, Les Temps/J.A. Lefebvre, 1914).

${ }^{*}$ Hémon, Louis, Maria Chapdelaine, Barcelona, Llibreria Catalònia, 1925. Réédité en 1952 chez Selecta; en 1984 et 1986 chez Proa. (Trad. de Tomàs Garcés. Original français: Maria Chapdelaine, Paris, Les Temps/J.A. Lefebvre, 1914).

LAFERRIÈRE, Dany, Cómo hacer el amor con un negro sin cansarse, Barcelona, Destino, 1997. (Traduction en espagnol de Lluís Maria Todó Vila. Original français: Comment faire l'amour avec un nègre sans se fatiguer, Montréal, VLB, 1995).

LAFERRIÈRE, Dany, ¿Esa granada en la mano del joven negro es un arma o una fruta?, Barcelona, Ed. Cobre, 2004 (Traduction de l'espagnol de Manuel Serrat Crespo. Original français: Cette grenade dans la main du jeune nègre est-elle une arme ou un fruit?, Montréal, VLB, 2002).

Lemieux, Jean, Los conquistadores del infinito, Madrid, Edelvives, 2003 (Traduction en espagnol de Elena del Amo. Original français: Les conquérants de l'infini, Montréal, La courte échelle, 2001).

Lemieux, Jean, Por tu bien, Madrid, Edelvives, 2004 (Traduction en espagnol de Elena del Amo. Original français: Le bonheur est une tempête avec un chien, Montréal, La courte échelle, 2001).

Marcotte, Gilles, Una misión difícil, Barcelona, Andrés Bello, 1998. (Traduction en espagnol de Alejandro Madrid-Zan. Original français: Une mission difficile, Montréal, Boréal, 1997).

${ }^{*}$ MarineaU, Michèle, Cassiopea o L'estiu polonès. Barcelona, La Galera, 1990. Réédité chez Columna en 1993. (Traduction en catalan de Álvar Valls. Original français: Cassiopée ou l'été polonais, Montréal, Éditions Québec/Amérique, 1988).

MarineaU, Michèle, Casiopea o el verano polaco, Barcelona, La Galera, 1991. (Traduction en espagnol de Angelina Gatell. Original français: Cassiopée ou l'été polonais, Montréal, Éditions Québec/Amérique, 1988). 
${ }^{*}$ MARTEL, Suzanne, Surreal 3000. Barcelona, Bruño, 1995. Réédité chez Bruño en 1996. (Traduction en catalan de Josep A. Vidal. Original français: Surréal 3000, Montréal, Héritage, 1989).

Ollivier, Émile, Pasos, Tafalla (Nafarroa), Txalaparta , 2004. (Traduction en espagnol de Rafael Yáñez Durán. Original français: Passages, Montréal, l'Hexagone, 1991).

${ }^{*}$ Plante, Raymond, La màquina de la bellesa, Barcelona, La Galera, S.A. Editorial, 1986. (Traduction en catalan de Àlvar Valls. Original français: La Machine à beauté, Montréal, Boréal, 1982).

Plante, Raymond, La máquina de la belleza, Barcelona, La Galera, S.A. Editorial 1986.

(Traduction en espagnol de Angelina Gatell. Original français: La Machine à beauté, Montréal, Boréal, 1982).

Plante, Raymond, El récord de Philibert Dupont, Barcelona, La Galera, S.A. Editorial, 1988. (Traduction en espagnol de Angelina Gatell. Original français: Le Record de Philibert Dupont, Montréal, Éditions Québec/Amérique, 1984).

${ }^{*}$ Plante, Raymond, El rècord d'en Philibert Dupont, Barcelona, La Galera, S.A. Editorial, 1988. (Traduction en catalan de Àlvar Valls. Original français: Le Record de Philibert Dupont, Éditions Québec/Amérique, 1984).

${ }^{*}$ Plante, Raymond, L’ultim estaquirot, Barcelona, La Galera, S.A. Editorial, 1989. Réédité en 1991 dans la même maison d'édition et chez Columna en 1993. (Traduction en catalan de Alvar Valls. Original français: Le Dernier des raisins, Montréal, Éditions Québec/Amérique, 1986).

Plante, Raymond, El último pasmarote, Barcelona, La Galera, S.A. Editorial 1990. (Traduction en espagnol de Angelina Gatell. Original français: Le Dernier des raisins, Montréal, Éditions Québec/Amérique, 1986).

${ }^{\star}$ Plante, Raymond, El gos salsitxa i els lladres de diamants, Barcelona, Édit. Núria Font i Ferré, 1992. (Traduction en catalan de Pau-Joan Hernández. Original français: Le Chien saucisse et les voleurs de diamants, Montréal, Boréal, 1991).

${ }^{*}$ Plante, Raymond, El rei de la salsitxa, Barcelona, La Galera, S.A. Editorial, 1992 (Traduction en catalan de Francesc Garriga. Original français: Des hot dogs sous le soleil, Montréal, Éditions Québec/Amérique, 1987)

SAvore, Jacques, La más popular del mundo, Zaragoza, Edelvives, 1999. (Traduction en espagnol de Susana Vázquez Jiménez. Original français: La plus populaire du monde, Montréal, La courte échelle, 1998)

SChinkel, David et Yves BeAuchesne, Ida y vuelta, León, Everest, 1999. (Traduction en espagnol de Ángel García Aller. Original français: Aller retour, Montréal, Pierre Tisseyre, 1986)

Schinkel, David et Yves Beauchesne, El don, León, Everest, 1999. (Traduction en espagnol de Ángel García Aller. Original français: Le don, Montréal, Pierre Tisseyre, 1990)

Soucy, Gaétan, La absolución, Barcelona, Andrés Bello, 1999 (Traduction en espagnol de Óscar Luis Molina. Original français: L’Acquittement, Montréal, Boréal, 1997).

Soucy, Gaétan, La niña que amaba las cerillas, Akal Literaria, 2001. (Traduction en espagnol de Oscar Luis Molina. Original français: La petite fille qui aimait trop les allumettes, Montréal, Boréal, 1997).

${ }^{\star}$ Soucy, Gaétan, La nena que li agradaven massa els llumins, Edit. Limits, 2001. (Traduction en catalan de Joan Casas. Original français: La petite fille qui aimait trop les allumettes, Montréal, Boréal, 1997).

Soucy, Gaétan, ; Music-hall!, Akal Literaria, 2004. (Traduction en espagnol de Esperanza Martínez. Original français: Music-hall!, Montréal, Boréal, 2002).

Soulières, Robert, Rompecabezas chino, Barcelona, Aliorna, 1988 (Traduction en espagnol de Caterina Molina. Original français: Casse-tête chinois, Montréal, Cercle du Livre de France, 1988).

${ }^{\star}$ Soulières, Robert, Trencaclosques xinès, Barcelona, Aliorna, 1988 (Traduction catalane de Araceli Bruc. Original français: Casse-tête chinois, Cercle du Livre de France, 1988). 
ТАLвот, Gilbert, Félix y Sofía, Barcelona, Aliorna, 1988 (Traduction et adaptation espagnole de Alicia Poza Sebastián. Original français: Félix et Sofia, Québec, Édition du Loup de goutière, 1992).

TrudeL, Sylvain, Los domingos de Julia, Madrid, Edelvives, 2001 (Traduction espagnole de Lourdes Huanqui. Original français: Les dimanches de Julie, Montréal, La courte échelle, 1998).

TrudeL, Sylvain, El niño que soñaba con ser héroe, Madrid, Edelvives, 2002 (Traduction et espagnole de P. Rozarena. Original français: Le garçon qui rêvait d'être un héros, Montréal, La courte échelle, 1995).

${ }^{\star}$ TrudeL, Sylvain. El nen que somiava ser heroi, Barcelona, Baula, 2003 (Traduction catalane de Elena Martín i Valls. Original français: Le garçon qui rêvait d'être un héros, Montréal, La courte échelle, 1995).

${ }^{*}$ TURCotTe, Élise, El soroll de les coses vives, Barcelona, La Magrana, 2001. (Traduction en catalan de Lourdes Bigorra. Original français: Le bruit des choses vivantes, Montréal, Leméac, 1991).

${ }^{\star}$ VANASSE, André, Milions per una cançó, Barcelona, La Galera, 1991 (Traduction catalane de Jacint Creus. Original français: Des millions pour une chanson, Montréal, Éditions Québec/ Amérique, 1988).

VANASSE, André, Millones por una canción, Barcelona, La Galera, 1992 (Traduction espagnole de Mercedes Caballud. Original français: Des millions pour une chanson, Montréal, Éditions Québec/Amérique, 1988). 